\title{
UNIVERSAL COVER OF SALVETTI'S COMPLEX AND TOPOLOGY OF SIMPLICIAL ARRANGEMENTS OF HYPERPLANES
}

\author{
LUIS PARIS
}

\begin{abstract}
Let $V$ be a real vector space. An arrangement of hyperplanes in $V$ is a finite set $\mathscr{A}$ of hyperplanes through the origin. A chamber of $\mathscr{A}$ is a connected component of $V-\left(\bigcup_{H \in \mathscr{A}} H\right)$. The arrangement $\mathscr{A}$ is called simplicial if $\bigcap_{H \in \mathscr{A}} H=\{0\}$ and every chamber of $\mathscr{A}$ is a simplicial cone. For an arrangement $\mathscr{A}$ of hyperplanes in $V$, we set
\end{abstract}

$$
M(\mathscr{A})=V_{\mathrm{C}}-\left(\bigcup_{H \in \mathscr{A}} H_{\mathrm{C}}\right),
$$

where $V_{\mathbf{C}}=\mathbb{C} \otimes V$ is the complexification of $V$, and, for $H \in \mathscr{A}, H_{\mathbb{C}}$ is the complex hyperplane of $V_{\mathrm{C}}$ spanned by $H$.

Let $\mathscr{A}$ be an arrangement of hyperplanes of $V$. Salvetti constructed a simplicial complex $\operatorname{Sal}(\mathscr{A})$ and proved that $\operatorname{Sal}(\mathscr{A})$ has the same homotopy type as $M(\mathscr{A})$. In this paper we give a new short proof of this fact. Afterwards, we define a new simplicial complex $\widehat{\operatorname{Sal}}(\mathscr{A})$ and prove that there is a natural map $p: \widehat{\operatorname{Sal}}(\mathscr{A}) \rightarrow \operatorname{Sal}(\mathscr{A})$ which is the universal cover of $\operatorname{Sal}(\mathscr{A})$. At the end, we use $\widehat{\operatorname{Sal}}(\mathscr{A})$ to give a new proof of Deligne's result: "if $\mathscr{A}$ is a simplicial arrangement of hyperplanes, then $M(\mathscr{A})$ is a $K(\pi, 1)$ space." Namely, we prove that $\widehat{\operatorname{Sal}}(\mathscr{A})$ is contractible if $\mathscr{A}$ is a simplicial arrangement.

\section{INTRODUCTION}

Let $V$ be a real vector space. An arrangement of hyperplanes in $V$ is a finite set $\mathscr{A}$ of hyperplanes through the origin. We say that $\mathscr{A}$ is essential if $\bigcap_{H \in \mathscr{A}} H=\{0\}$. A chamber of $\mathscr{A}$ is a connected component of $V-\bigcup_{H \in \mathscr{A}} H$. The arrangement $\mathscr{A}$ is called simplicial if $\mathscr{A}$ is essential and every chamber of $\mathscr{A}$ is an open simplicial cone.

Let $V_{\mathbb{C}}=\mathbb{C} \otimes V$ be the complexification of $V$. Every element $z$ of $V_{\mathbb{C}}$ can be written in a unique way $z=x+i y$, where $x, y \in 1 \otimes V=V$. We say that $x$ is the real part of $z$ and that $y$ is its imaginary part. For two subsets $X, Y \subseteq V$, we write

$$
X+i Y=\left\{(x+i y) \in V_{\mathbb{C}} \mid x \in X \text { and } y \in Y\right\} .
$$

Let $H$ be a hyperplane of $V$. The complexification $H_{\mathbb{C}}$ of $H$ is the hyperplane of $V_{\mathbb{C}}$ spanned by $H ; H_{\mathbb{C}}=H+i H$.

Received by the editors June 25, 1991.

1991 Mathematics Subject Classification. Primary 52B30.

Supported by the FNSRS (Swiss National Foundation). 
Let $\mathscr{A}$ be an arrangement of hyperplanes in a real vector space $V$. We set

$$
M(\mathscr{A})=V_{\mathbb{C}}-\left(\bigcup_{H \in \mathscr{A}} H_{\mathbb{C}}\right) .
$$

This space is an open connected submanifold of $V_{\mathbb{C}}$.

The study of the topology of $M(\mathscr{A})$ can be easily reduced to the case of an essential arrangement of hyperplanes. Thus we assume throughout all the arrangements to be essential.

In [Sa1], Salvetti associates with a real arrangement $\mathscr{A}$ of hyperplanes a simplicial complex $\operatorname{Sal}(\mathscr{A})$, and proves that $\operatorname{Sal}(\mathscr{A})$ has the same homotopy type as $M(\mathscr{A})$.

An oriented system is a pair $(\Gamma, \sim)$ where $\Gamma$ is an oriented graph and $\sim$ is an equivalence relation on the set of paths of $\Gamma$ with some properties described in $\S 2$. In [Pa1], the author shows that there is a natural notion of universal cover $\rho:(\widehat{\Gamma}, \sim) \rightarrow(\Gamma, \sim)$ of an oriented system $(\Gamma, \sim)$, associates an oriented system $(\Gamma(\mathscr{A}), \sim)$ with a real arrangement $\mathscr{A}$ of hyperplanes, and constructs, from the universal cover of $(\Gamma(\mathscr{A}), \sim)$, the universal cover of $M(\mathscr{A})$.

Note that, in [Sa1], Salvetti uses this same oriented system $(\Gamma(\mathscr{A}), \sim)$ to compute the fundamental group of $M(\mathscr{A})$. He also proves that $\Gamma(\mathscr{A})$ is the 1 -skeleton of $\operatorname{Sal}(\mathscr{A})$ provided with an orientation, and that $\sim$ is the homotopy relation on the paths of $\Gamma(\mathscr{A})$. Nevertheless, both works, [Sa1] and [Pa1], are completely independent.

In this paper we give a new short proof of Salvetti's result: $\operatorname{Sal}(\mathscr{A})$ has the same homotopy type as $M(\mathscr{A})$ (Theorem 3.3). Afterwards, using techniques introduced in [Pa1], we define another simplicial complex $\widehat{\mathrm{Sal}}(\mathscr{A})$, and prove that there is a natural map $p: \widehat{\operatorname{Sal}}(\mathscr{A}) \rightarrow \operatorname{Sal}(\mathscr{A})$ which is the universal cover of $\operatorname{Sal}(\mathscr{A})$ (Theorem 3.7). In particular, $\widehat{\operatorname{Sal}}(\mathscr{A})$ has the same homotopy type as the universal cover of $M(\mathscr{A})$. At the end, we use $\widehat{\operatorname{Sal}}(\mathscr{A})$ to give a new proof of the following result of Deligne.

Theorem 1.1 (Deligne [De]). If $\mathscr{A}$ is a simplicial arrangement of hyperplanes, then $M(\mathscr{A})$ is a $K(\pi, 1)$ space (i.e., the universal cover of $M(\mathscr{A})$ is contractible).

Namely, we prove that $\widehat{\operatorname{Sal}}(\mathscr{A})$ is contractible if $\mathscr{A}$ is a simplicial arrangement.

We say that a real arrangement $\mathscr{A}$ of hyperplanes is a $K(\pi, 1)$ arrangement if $M(\mathscr{A})$ is a $K(\pi, 1)$ space. Most of the already known $K(\pi, 1)$ arrangements are supersolvable (see [Te2]) or simplicial. To find a general criterion for an arrangement to be $K(\pi, 1)$ remains an open problem. In particular, Saito's conjecture that free arrangements are $K(\pi, 1)$ is unsolved (see [Te1]). Supersolvable arrangements are free (see [JT]) but simplicial arrangements are not always free (see [Te1]). We refer to [FR] for a good exposition on $K(\pi, 1)$ arrangements.

The interest of our simplicial complex $\widehat{\operatorname{Sal}}(\mathscr{A})$ is, in order to prove that a real arrangement $\mathscr{A}$ is $K(\pi, 1)$, it suffices to prove that $\widehat{\operatorname{Sal}}(\mathscr{A})$ is contractible.

Our proof of Theorem 1.1 is more simple than Deligne's one and, more- 
over, we isolate the part of the proof where the hypothesis " $\mathscr{A}$ is a simplicial arrangement of hyperplanes" is essential.

For a simplicial arrangement $\mathscr{A}$ of hyperplanes, Deligne constructs in [De] a cover $q: \widehat{M} \rightarrow M(\mathscr{A})$, defines a simplicial complex $\operatorname{Del}(\mathscr{A})$, and proves that $\operatorname{Del}(\mathscr{A})$ is contractible and has the same homotopy type as $\widehat{M}$. In particular, $\widehat{M}$ is the universal cover of $M(\mathscr{A})$.

Our first innovation was in [Pal] to introduce a new combinatorial tool: the oriented system. Deligne's combinatorial tool, the groupoid Gal, is, in some sense, equivalent to our oriented system, but it cannot be defined for any real arrangement of hyperplanes ((i) and (ii) of Proposition 1.19 of [De] are needed to define Gal and their proof strongly uses the fact that $\mathscr{A}$ is simplicial), and, moreover, unlike in oriented systems, several preliminary results are needed to define it (in particular, Ore's criterion for a semigroup to be embedded in a group (see [Lj, p. 400]) must be generalized to "semigroupoids" and groupoids).

Another important innovation is to substitute the simplicial complex $\operatorname{Del}(\mathscr{A})$ for $\widehat{\operatorname{Sal}}(\mathscr{A})$. Using oriented systems, the complex $\operatorname{Del}(\mathscr{A})$ can be generalized to any real arrangement of hyperplanes (in the general case, $\operatorname{Del}(\mathscr{A})$ is a $\mathrm{CW}$ complex), but it does not always have the same homotopy type as the universal cover $\widehat{M}$ of $M(\mathscr{A})$ (see [Pa2]). An advantage of our complex $\widehat{\operatorname{Sal}}(\mathscr{A})$ over $\operatorname{Del}(\mathscr{A})$ is, in order to prove that $\operatorname{Del}(\mathscr{A})$ has the same homotopy type as $\widehat{M}$, several considerations on some kind of "subgroupoids" of $\mathrm{Gal}$ are needed (see (1.5), (1.6), (1.9), (1.24), (1.28), (1.29), (1.30), (1.31), (1.32) of [De]) which are not necessary in our case.

To prove that either $\widehat{\operatorname{Sal}}(\mathscr{A})$ or $\operatorname{Del}(\mathscr{A})$ are contractible, a strong property of simplicial arrangements is needed: the property $D$ (see Theorem 4.1). In our proof of Theorem 1.1 the hypothesis " $\mathscr{A}$ is a simplicial arrangement of hyperplanes" is only used to show that $\mathscr{A}$ has the property D. In fact, for an essential arrangement $\mathscr{A}$ of hyperplanes, having the property $\mathrm{D}$ is equivalent to being simplicial. I actually proved it some time after the first version of this paper (see $[\mathrm{Pa} 3])$.

The only place where our proof of Theorem 1.1 coincides with Deligne's one is in our Lemma 4.4. It is a preliminary result to the proof of Theorem 4.1.

Since I submitted for publication the first version of my paper, Raul Cordovil has informed me he has independently of my paper generalized Deligne's theorem for simplicial oriented matroids (see [Co]). On the other hand, Mario Salvetti has informed me he has also proved independently Theorem 4.1 and Theorem 4.6 (see [Sa2]).

Our work is organized as follows.

Section 2 is a summary of [ $\mathrm{Pa} 1]$. Its aim is to introduce our main combinatorial tool, the oriented systems, and to give the construction of the universal cover $\widehat{M} \rightarrow M(\mathscr{A})$ of $M(\mathscr{A})$.

In $\S 3$ we define the simplicial complexes $\operatorname{Sal}(\mathscr{A})$ and $\widehat{\operatorname{Sal}}(\mathscr{A})$, and prove that $\operatorname{Sal}(\mathscr{A})$ has the same homotopy type as $M(\mathscr{A})$, and that there is a natural map $p: \widehat{\operatorname{Sal}}(\mathscr{A}) \rightarrow \operatorname{Sal}(\mathscr{A})$ which is the universal cover of $\operatorname{Sal}(\mathscr{A})$.

The goal of $\S 4$ is to prove Theorem 1.1.

I am grateful to Peter Orlik and Hiroaki Terao who have helped me with discussions, suggestions, and encouragement during my work. 


\section{THE UNIVERSAL COVER OF $M(\mathscr{A})$}

This section is divided into three subsections. In the first one we introduce our main combinatorial tool: the oriented system. In the second subsection we define the oriented system $(\Gamma(\mathscr{A}), \sim)$ associated with a real arrangement $\mathscr{A}$ of hyperplanes. In the third subsection, from the universal cover $\rho:(\widehat{\Gamma}, \sim) \rightarrow$ $(\Gamma(\mathscr{A}), \sim)$ of the oriented system $(\Gamma(\mathscr{A}), \sim)$, we give the construction of the universal cover $\widehat{M} \rightarrow M(\mathscr{A})$ of $M(\mathscr{A})$.

All the results stated in this section are derived from [Pa1], so we will not give any proofs.

2A. Oriented systems. An oriented graph $\Gamma$ is the following data:

(1) a set $V(\Gamma)$ of vertices,

(2) a subset $A(\Gamma) \subseteq(V(\Gamma) \times V(\Gamma))-\{(v, v) \mid v \in V(\Gamma)\}$ of arrows.

The begin of an arrow $a=(v, w)$ is $v$ and its end is $w$. An oriented graph $\Gamma$ is locally finite if every vertex $v \in V(\Gamma)$ is the begin or the end of only a finite number of arrows.

A path of an oriented graph $\Gamma$ is an expression

$$
f=a_{1}^{\varepsilon_{1}} a_{2}^{\varepsilon_{2}} \cdots a_{n}^{\varepsilon_{n}},
$$

where $a_{i} \in A(\Gamma)$ and $\varepsilon_{i} \in\{ \pm 1\}$ (for $\left.i=1, \ldots, n\right)$, such that there exists a sequence $v_{0}, v_{1}, \ldots, v_{n}$ of vertices of $\Gamma$ with

$$
\begin{array}{ll}
a_{i}=\left(v_{i-1}, v_{i}\right) & \text { if } \varepsilon_{i}=1, \\
a_{i}=\left(v_{i}, v_{i-1}\right) & \text { if } \varepsilon_{i}=-1 .
\end{array}
$$

We say that $v_{0}$ is the begin of $f$ and that $v_{n}$ is its end. The integer $n$ is its length and $\sum_{i=1}^{n} \varepsilon_{i}$ is its weight. Every vertex of $\Gamma$ is assumed to be a path of length 0 and of weight 0 . For a path $f=a_{1}^{\varepsilon_{1}} \cdots a_{n}^{\varepsilon_{n}}$, we write $f^{-1}=$ $a_{n}^{-\varepsilon_{n}} \cdots a_{1}^{-\varepsilon_{1}}$. For two paths $f=a_{1}^{\varepsilon_{1}} \cdots a_{n}^{\varepsilon_{n}}$ and $g=b_{1}^{\mu_{1}} \cdots b_{m}^{\mu_{m}}$ with end $(f)=$ begin $(g)$, we write $f g=a_{1}^{\varepsilon_{1}} \ldots a_{n}^{\varepsilon_{n}} b_{1}^{\mu_{1}} \ldots b_{m}^{\mu_{m}}$.

An oriented graph $\Gamma$ is connected if, for every pair $(v, w)$ of vertices of $\Gamma$, there exists a path of $\Gamma$ which begins at $v$ and ends in $w$.

We assume throughout all the oriented graphs to be locally finite and connected.

Let $\Gamma$ be an oriented graph. An identification of $\Gamma$ is an equivalence relation $\sim$ in the set of paths of $\Gamma$ with the following properties:

(1) $f \sim g \Rightarrow \operatorname{begin}(f)=\operatorname{begin}(g)$, end $(f)=\operatorname{end}(g)$, and weight $(f)=$ weight $(g)$,

(2) $f f^{-1} \sim$ begin $(f)$, for every path $f$,

(3) $f \sim g \Rightarrow f^{-1} \sim g^{-1}$,

(4) $f \sim g \Rightarrow h_{1} f h_{2} \sim h_{1} g h_{2}$ for any two paths $h_{1}$ and $h_{2}$ such that $\operatorname{end}\left(h_{1}\right)=\operatorname{begin}(f)=\operatorname{begin}(g)$ and $\operatorname{begin}\left(h_{2}\right)=\operatorname{end}(f)=\operatorname{end}(g)$.

An oriented system is a pair $(\Gamma, \sim)$, where $\Gamma$ is an oriented graph and $\sim$ is an identification of $\Gamma$.

Let $\rho: \Theta \rightarrow \Gamma$ be a morphism of oriented graphs. We say that $\rho$ is a cover of $\Gamma$ if, for every vertex $v$ of $\Theta$ and every path $f$ of $\Gamma$ beginning at $\rho(v)$, there exists a unique path $\hat{f}$ of $\Theta$ such that begin $(\hat{f})=v$ and $\rho(\hat{f})=f$. 
Let $\rho:(\Theta, \sim) \rightarrow(\Gamma, \sim)$ be a morphism of oriented systems (i.e., $\hat{f} \sim \hat{g} \Rightarrow$ $\rho(\hat{f}) \sim \rho(\hat{g}))$. We say that $\rho$ is a cover of $(\Gamma, \sim)$ if it has the following two properties:

(1) $\rho: \Theta \rightarrow \Gamma$ is a cover of $\Gamma$.

(2) Let $v \in V(\Theta)$, let $f$ and $g$ be two paths of $\Gamma$ which both begin at $\rho(v)$, and let $\hat{f}$ and $\hat{g}$ be the lifts of $f$ and $g$ respectively into $\Theta$ beginning at $v$. If $f \sim g \quad(\Rightarrow \operatorname{end}(f)=\operatorname{end}(g))$, then $\hat{f} \sim \hat{g} \quad(\Rightarrow \operatorname{end}(\hat{f})=\operatorname{end}(\hat{g}))$.

Example. Let $\Gamma$ be the oriented graph shown in Figure 1a. Let $\sim$ be the smallest identification of $\Gamma$ such that $a b \sim d c$. The identification $\sim$ can be viewed as a "homotopy relation", namely, to identify $a b$ with $d c$ is like to "add" a 2-cell to $\Gamma$ having $a b c^{-1} d^{-1}$ as border. Let $\widehat{\Gamma}$ be the oriented graph shown in Figure 1b. The morphism $\pi: \widehat{\Gamma} \rightarrow \Gamma$ which sends $a_{i}$ onto $a, b_{i}$ onto $b, c_{i}$ onto $c, d_{i}$ onto $d$, and $e_{i}$ onto $e$ (where $i \in \mathbb{Z}$ ) is obviously a cover of $\Gamma$. Let $\sim$ be the smallest identification of $\widehat{\Gamma}$ such that $a_{i} b_{i} \sim d_{i} c_{i}$ for every $i \in \mathbb{Z}$. The map $\pi:(\widehat{\Gamma}, \sim) \rightarrow(\Gamma, \sim)$ is a cover of $(\Gamma, \sim)$; in fact, it is the universal cover of $(\Gamma, \sim)$ (see Proposition 2.1).

Proposition 2.1. Let $(\Gamma, \sim)$ be an oriented system. There exists a unique cover $\pi:(\widehat{\Gamma}, \sim) \rightarrow(\Gamma, \sim)$ of $(\Gamma, \sim)$ (up to isomorphism) which has the following universal property. If $\rho:(\Theta, \sim) \rightarrow(\Gamma, \sim)$ is a cover of $(\Gamma, \sim)$, then there exists a unique cover $\pi^{\prime}:(\widehat{\Gamma}, \sim) \rightarrow(\Theta, \sim)$ of $(\Theta, \sim)$ (up to isomorphism) such that $\pi=\rho \circ \pi^{\prime}$.

We call $\pi:(\widehat{\Gamma}, \sim) \rightarrow(\Gamma, \sim)$ the universal cover of $(\Gamma, \sim)$.

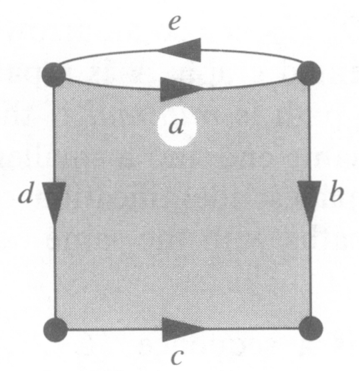

Figure 1a

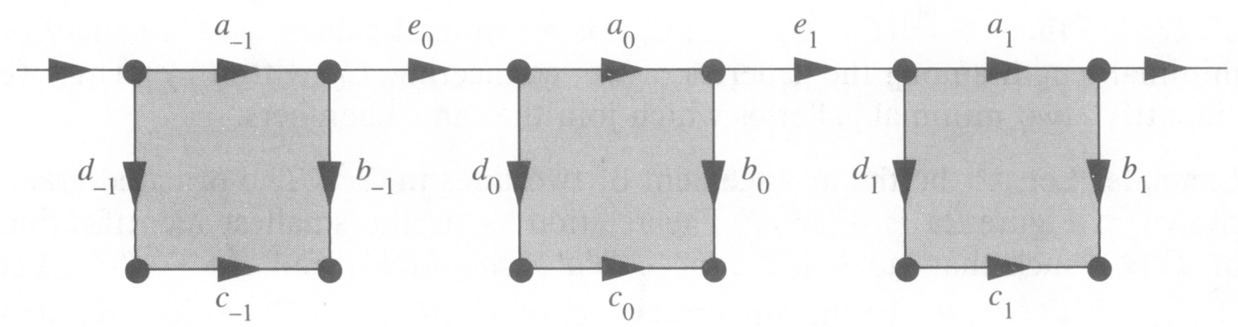

Figure 1b 
Proposition 2.2. Let $\pi:(\widehat{\Gamma}, \sim) \rightarrow(\Gamma, \sim)$ be the universal cover of an oriented system $(\Gamma, \sim)$. Two paths $\hat{f}$ and $\hat{g}$ of $\hat{\Gamma}$ are identified by $\sim$ if and only if $\operatorname{begin}(\hat{f})=\operatorname{begin}(\hat{g})$ and $\operatorname{end}(\hat{f})=\operatorname{end}(\hat{g})$.

2B. Definition of $(\Gamma(\mathscr{A}), \sim)$. Let $\mathscr{A}$ be an (essential) arrangement of hyperplanes in a real vector space $V$. Our goal in this subsection is to associate with $\mathscr{A}$ an oriented system $(\Gamma(\mathscr{A}), \sim)$.

First, recall some definitions. The lattice of $\mathscr{A}$ is the poset

$$
\mathscr{L}(\mathscr{A})=\left\{\bigcap_{H \in \mathscr{B}} H \mid \mathscr{B} \subseteq \mathscr{A}\right\},
$$

ordered by reverse inclusion. $V=\bigcap_{H \in \varnothing} H$ is the smallest element of $\mathscr{L}(\mathscr{A})$ and $\{0\}=\bigcap_{H \in \mathscr{A}} H$ is the greatest one. For $X \in \mathscr{L}(\mathscr{A})$, we set

$$
\mathscr{A}_{X}=\{H \in \mathscr{A} \mid H \supseteq X\} .
$$

We refer to [Or] for a good exposition on $\mathscr{L}(\mathscr{A})$ and its properties.

The hyperplanes of $\mathscr{A}$ subdivide $V$ into facets. We denote by $\mathscr{F}(\mathscr{A})$ the set of all the facets. The support $|F|$ of a facet $F$ is the vector space $|F| \in \mathscr{L}(\mathscr{A})$ spanned by $F$. Every facet is open in its support. We denote by $\bar{F}$ the closure of $F$ in $V$. There is a partial order in $\mathscr{F}(\mathscr{A})$ defined by $F \leq G$ if $F \subseteq \bar{G}$.

A chamber of $\mathscr{A}$ is a 0 codimension facet. A face is a 1 codimension facet. Two chambers $C$ and $D$ are adjacent if they have a common face (i.e., a common 1 codimension facet).

Now, let us define the oriented system $(\Gamma, \sim)=(\Gamma(\mathscr{A}), \sim)$ associated with $\mathscr{A}$.

The vertices of $\Gamma$ are the chambers of $\mathscr{A}$. An arrow of $\Gamma$ is a pair $(C, D)$, where $C$ and $D$ are adjacent chambers. Note that, in this oriented graph, if $(C, D)$ is an arrow, then $(D, C)$ is also an arrow.

A positive path of an oriented graph $\Delta$ is a path $f=a_{1}^{\varepsilon_{1}} \cdots a_{n}^{\varepsilon_{n}}$ with $\varepsilon_{1}=$ $\cdots=\varepsilon_{n}=1$. This positive path is minimal if there is no positive path in $\Delta$ having the same begin, the same end and a smaller length than $f$.

The relation $\sim$ is the smallest identification of $\Gamma$ such that if $f$ and $g$ are both positive minimal paths with the same begin and the same end, then $f \sim g$.

Remark. A gallery of $\mathscr{A}$ is a sequence $\left(C_{0}, C_{1}, \ldots, C_{n}\right)$ of chambers of $\mathscr{A}$ such that $C_{i-1}$ and $C_{i}$ are adjacent for $i=1, \ldots, n$ (here we assume $C_{i-1} \neq C_{i}$ for $\left.i=1, \ldots, n\right)$. Any positive path $f=a_{1} \cdots a_{n}$ of $\Gamma(\mathscr{A})$ can be viewed as the gallery $G=\left(C_{0}, C_{1}, \ldots, C_{n}\right)$, where $C_{i}=\operatorname{end}\left(a_{1} \cdots a_{i}\right)$ for $i=0,1, \ldots, n$. In particular, if $f=a_{1} \cdots a_{n}$ is a positive minimal path of $\Gamma(\mathscr{A})$, then $G=\left(C_{0}, C_{1}, \ldots, C_{n}\right)$ is a minimal gallery (i.e., a gallery of minimal length among the galleries of $\mathscr{A}$ connecting $C_{0}$ with $C_{n}$ ). Thus we "identify" two minimal galleries which join the same chambers.

Example. Let $\mathscr{A}$ be the arrangement of two lines in $\mathbb{R}^{2}$. The oriented graph shown in Figure $2 \mathrm{a}$ is $\Gamma(\mathscr{A})$. The relation $\sim$ is the smallest identification of $\Gamma(\mathscr{A})$ such that $a b \sim a^{\prime} d^{\prime}, b c \sim b^{\prime} a^{\prime}, c d \sim c^{\prime} b^{\prime}$, and $d a \sim d^{\prime} c^{\prime}$. Let $(\widehat{\Gamma}, \sim) \rightarrow(\Gamma(\mathscr{A}), \sim)$ be the universal cover of $(\Gamma(\mathscr{A}), \sim)$. The oriented graph shown in Figure $2 b$ is $\hat{\Gamma}$. 


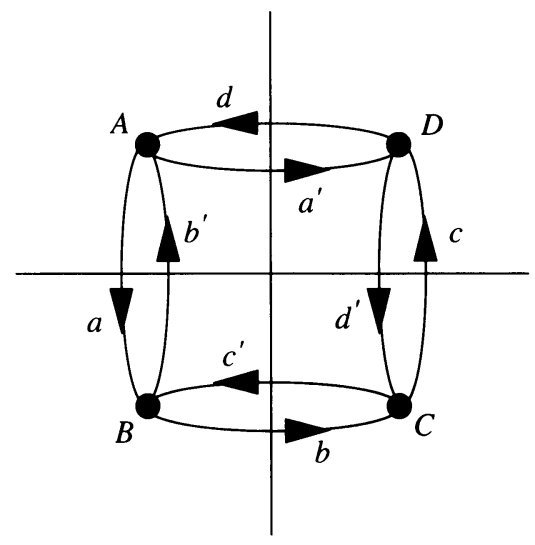

FIGURE 2a

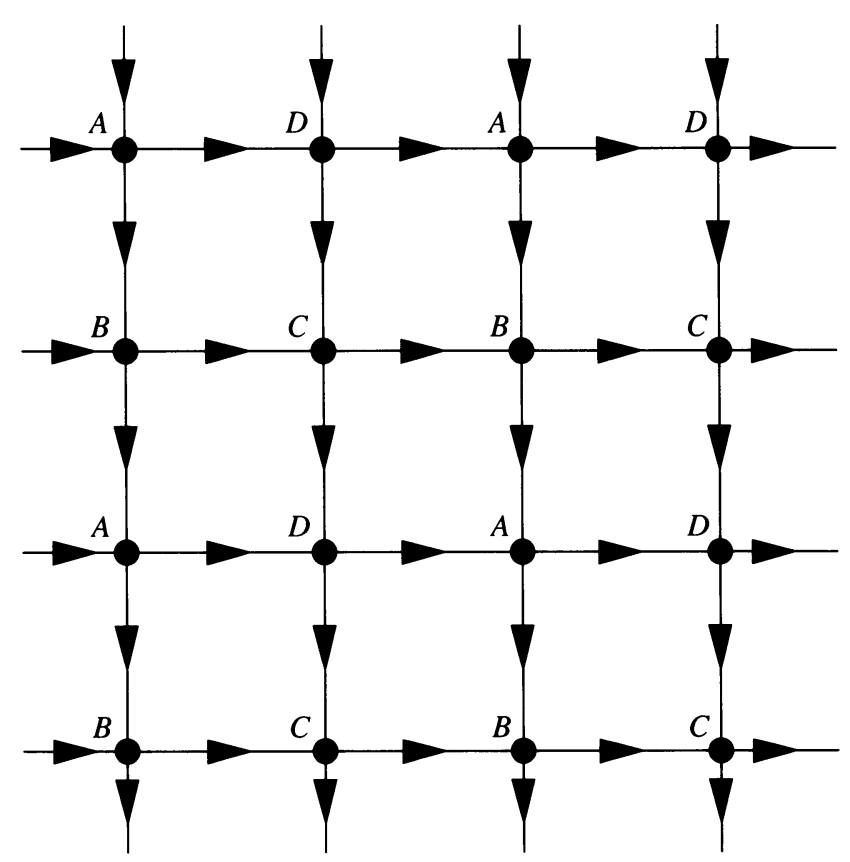

Figure 2b

2C. Universal cover of $M(\mathscr{A})$. Let $\mathscr{A}$ be an (essential) arrangement of hyperplanes in a real vector space $V$. We set

$$
M=M(\mathscr{A})=V_{\mathbb{C}}-\left(\bigcup_{H \in \mathscr{A}} H_{\mathbb{C}}\right)
$$

Our goal in this subsection is to explain the construction of the universal cover $q: \widehat{M} \rightarrow M$ of $M(\mathscr{A})$.

Let $C$ be a chamber of $\mathscr{A}$. For a facet $F \in \mathscr{F}(\mathscr{A})$, we denote by $C_{F}$ the 
unique chamber of $\mathscr{A}_{|F|}$ containing $C$. We write

$$
M(C)=\bigcup_{F \in \mathscr{F}(\mathscr{A})}\left(F+i C_{F}\right) \subseteq(V+i V)=V_{\mathbb{C}} .
$$

Note that this union is disjoint.

Lemma 2.3. The set $\{M(C) \mid C \in V(\Gamma)\}$ is a covering of $M(\mathscr{A})$ by open subsets. Proof. First, let us prove that $M(C) \subseteq M(\mathscr{A})$ for every chamber $C$ of $\mathscr{A}$.

Fix a chamber $C$ of $\mathscr{A}$. Pick $F \in \mathscr{F}(\mathscr{A})$. Let $H$ be a hyperplane of $\mathscr{A}$. If $H \supseteq F$, then $C_{F} \cap H=\varnothing$ (since $C_{F}$ is a chamber of $\mathscr{A}_{|F|}$ ), thus $\left(F+i C_{F}\right) \cap H_{\mathbb{C}}=\varnothing$. If $H \nsupseteq F$, then $F \cap H=\varnothing$, thus $\left(F+i C_{F}\right) \cap H_{\mathbb{C}}=\varnothing$. Therefore $\left(F+i C_{F}\right) \subseteq M(\mathscr{A})$ for every $F \in \mathscr{F}(\mathscr{A})$, thus $M(C) \subseteq M(\mathscr{A})$.

Now, let us prove that $M(C)$ is an open subset of $V_{\mathbb{C}}$ for every chamber $C$ of $\mathscr{A}$.

Fix a chamber $C$ of $\mathscr{A}$. Pick $z=(x+i y) \in M(C)$. Let $F$ be the facet of $\mathscr{A}$ such that $x \in F$. Then, by the definition of $M(C)$, we have $y \in C_{F}$. If $G$ is a facet of $\mathscr{A}$ with $G \geq F$, then $\mathscr{A}_{|F|} \supseteq \mathscr{A}_{|G|}$, thus $C_{F} \subseteq C_{G}$. We write $U(z)=\left(\bigcup_{G \geq F} G\right)+i C_{F}$. The set $U(z)$ is clearly an open neighbourhood of $z$ and, by the above considerations, $U(z) \subseteq M(C)$.

Now, let us prove that $M(\mathscr{A}) \subseteq \bigcup_{C \in V(\Gamma)} M(C)$.

Pick $z=(x+i y) \in M(\mathscr{A})$. Let $F$ be the facet of $\mathscr{A}$ such that $x \in F$. Then there is a chamber $D$ of $\mathscr{A}_{|F|}$ such that $y \in D$. Choose a chamber $C$ of $\mathscr{A}$ such that $C_{F}=D$. Then $z=(x+i y) \in\left(F+i C_{F}\right) \subseteq M(C)$.

Now, consider the universal cover $\rho:(\widehat{\Gamma}, \sim) \rightarrow(\Gamma(\mathscr{A}), \sim)$ of $(\Gamma(\mathscr{A}), \sim)$. For every vertex $v$ of $\widehat{\Gamma}$, write

$$
M(v)=M(\rho(v)) .
$$

Set

$$
M^{\prime}=\coprod_{v \in V(\widehat{\Gamma})} M(v),
$$

and let $q^{\prime}: M^{\prime} \rightarrow M$ be the natural projection.

It is easy to see that, if two chambers $C$ and $D$ are adjacent, then there is only one hyperplane $H \in \mathscr{A}$ which separates $C$ and $D$; it is the support of their common face. For a chamber $C$ of $\mathscr{A}$ and a hyperplane $H \in \mathscr{A}$, we denote by $H_{C}^{+}$the open half-space of $V$ bordered by $H$ and containing $C$.

Let $\mathscr{R}$ be the smallest equivalence relation on $M^{\prime}$ such that if $a=(v, w) \in$ $A(\widehat{\Gamma}), z \in M(v), z^{\prime} \in M(w)$, and

$$
q^{\prime}(z)=q^{\prime}\left(z^{\prime}\right) \in M(v) \cap M(w) \cap\left(H_{\rho(w)}^{+}+i V\right),
$$

where $H$ is the unique hyperplane of $\mathscr{A}$ which separates $\rho(v)$ and $\rho(w)$, then $z \mathscr{R} z^{\prime}$. The space $\widehat{M}$ is the quotient $\widehat{M}=M^{\prime} / \mathscr{R}$, and $q: \widehat{M} \rightarrow M$ is the map induced by $q^{\prime}$.

Theorem 2.4. The map $q: \widehat{M} \rightarrow M$ is the universal cover of $M(\mathscr{A})$.

Example. Let $V=\mathbb{R}$ and let $\mathscr{A}=\{0\}$. The chambers of $\mathscr{A}$ are $C=\{x \in$ $\mathbb{R} \mid x>0\}$ and $D=\{x \in \mathbb{R} \mid x<0\}$. The oriented graph shown in Figure 3a is $\Gamma(\mathscr{A})$ and the oriented graph shown in Figure $3 b$ is $\widehat{\Gamma}$. The subsets of $\mathbb{C}$ 
shown in Figure $3 c$ are $M(C)$ and $M(D)$. We have $M(C)=\mathbb{C}-\{i y \mid y \leq 0\}$ and $M(D)=\mathbb{C}-\{i y \mid y \geq 0\}$. Let $(v, w)$ be an arrow of $\widehat{\Gamma}$ with $\rho(v)=C$ and $\rho(w)=D$. We have

$$
\begin{aligned}
M(v) & \cap M(w) \cap\left(H_{\rho(w)}^{+}+i V\right) \\
= & (\mathbb{C}-\{i y \mid y \leq 0\}) \cap(\mathbb{C}-\{i y \mid y \geq 0\}) \cap\{(x+i y) \in \mathbb{C} \mid x<0\} \\
= & \{(x+i y) \in \mathbb{C} \mid x<0\} .
\end{aligned}
$$

The space shown in Figure $3 \mathrm{~d}$ is $(M(v) \amalg M(w)) / \mathscr{R}$. If we extend this construction to all $\widehat{\Gamma}$, then we clearly obtain the universal cover of $M(\mathscr{A})=\mathbb{C}-\{0\}$.

Lemmas 2.5, 2.6 and 2.7 are in [Pa1] preliminary results to the proof of Theorem 2.4; nevertheless, we state them since they will be used later in the paper.

Fix a vertex $v \in V(\widehat{\Gamma})$. Write $C=\rho(v)$. For every chamber $D$ of $\mathscr{A}$, we choose a positive minimal path $f_{D}$ of $\Gamma(\mathscr{A})$ beginning at $C$ and ending in $D$.We denote by $\hat{f}_{D}$ the lift of $f_{D}$ into $\widehat{\Gamma}$ beginning at $v$. Note that the end of $\hat{f}_{D}$ does not depend on the choice of $f_{D}$ (see the definition of the identification $\sim$ of $\Gamma$ ). We set

$$
\Sigma(v)=\left\{\operatorname{end}\left(\hat{f}_{D}\right) \mid D \in V(\Gamma)\right\}
$$

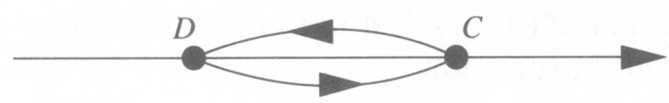

FIGURE 3a

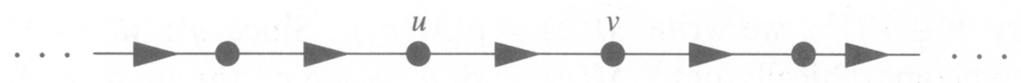

FIGURE 3b

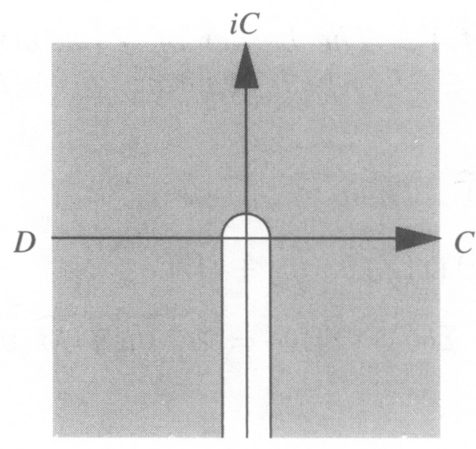

$i D$

$M(C)$

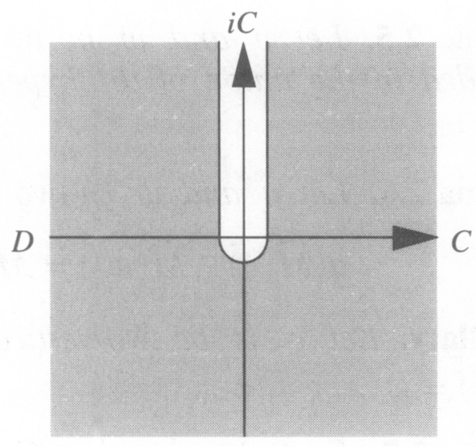

$i D$

$M(D)$

Figure 3c 


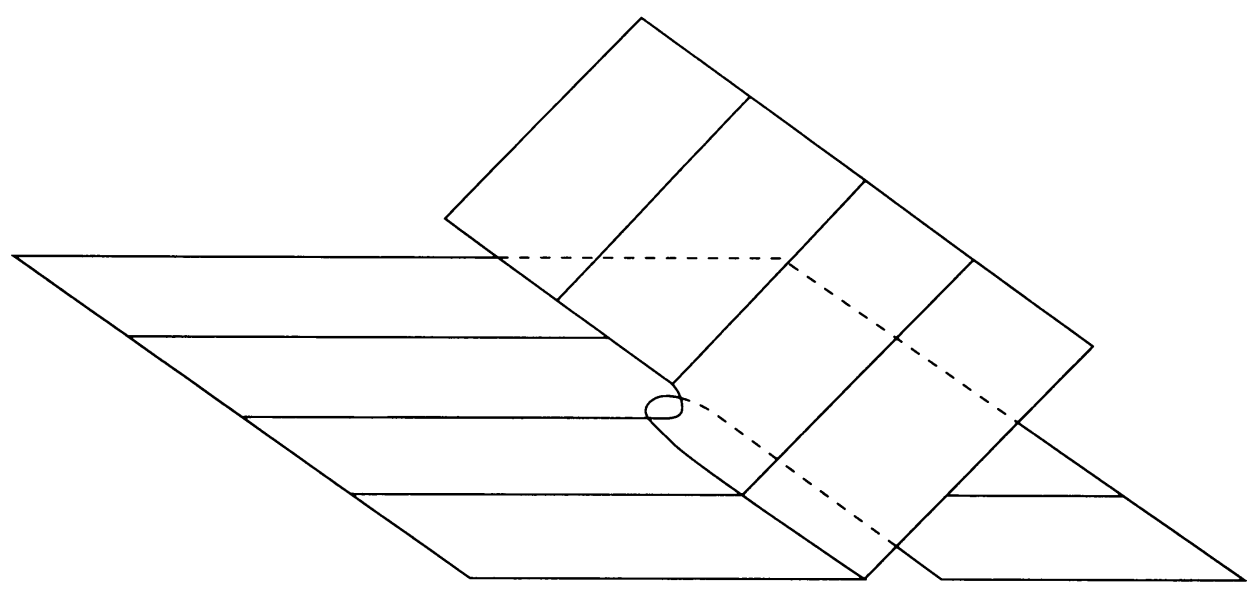

FIGURE 3d

The restriction of $\rho$ to $\Sigma(v)$ is clearly a bijection $\Sigma(v) \rightarrow V(\Gamma)$.

Let $v$ and $w$ be two vertices of $\widehat{\Gamma}$. We write

$$
\bar{Z}(v, w)=\bigcup_{u} \bar{\rho}(u),
$$

where the union is over al the vertices $u \in \Sigma(v) \cap \Sigma(w)$ and, for $u \in \Sigma(v) \cap \Sigma(w)$, the set $\bar{\rho}(u)$ is the closure of $\rho(u)$ in $V$. We denote by $Z(v, w)$ the interior of $\bar{Z}(v, w)$. Note that $Z(v, w)$ is a union of facets of $\mathscr{A}$.

Consider the natural projection

$$
p: M^{\prime}=\coprod_{v \in V(\widehat{\Gamma})} M(v) \rightarrow \widehat{M} .
$$

For every $v \in V(\widehat{\Gamma})$, we write $\widehat{M}(v)=p(M(v))$. Since $q^{\prime}: M^{\prime} \rightarrow M$ sends $M(v)$ homeomorphically onto $M(v)$ and $q^{\prime}=q \circ p$, the map $q: \widehat{M} \rightarrow M$ sends $\widehat{M}(v)$ homeomorphically onto $M(v)$. Moreover, since $q$ is a cover, $\widehat{M}(v)$ is an open subset of $\widehat{M}$.

Lemma 2.5. Let $v$ and $w$ be two vertices of $\widehat{\Gamma}$. The border of $Z(v, w)$ is included in the union of the hyperplanes $H \in \mathscr{A}$ which separate $\rho(v)$ and $\rho(w)$.

Lemma 2.6. Let $v$ and $w$ be two vertices of $\widehat{\Gamma}$. Then

$$
q(\widehat{M}(v) \cap \widehat{M}(w))=M(v) \cap M(w) \cap(Z(v, w)+i V) .
$$

Corollary. Let $v, w$ be two vertices of $\widehat{\Gamma}$. If $\Sigma(v) \cap \Sigma(w)=\varnothing$, then $\widehat{M}(v) \cap$ $\widehat{M}(w)=\varnothing$.

Lemma 2.7. For every chamber $C$ of $\mathscr{A}$, we have

$$
q^{-1}(M(C))=\bigcup_{v \in \rho^{-1}(C)} \widehat{M}(v),
$$

and this union is disjoint. 


\section{UNIVERSAl COVER OF SALVETti's COMPLEX}

We assume throughout this section $\mathscr{A}$ to be an (essential) arrangement of hyperplanes in a real vector space $V$ of dimension $l$.

This section is divided in two subsections. In the first one we define an (abstract) simplicial complex $\operatorname{Sal}(\mathscr{A})$ (Salvetti's complex) and prove that $\operatorname{Sal}(\mathscr{A})$ has the same homotopy type as $M(\mathscr{A})$. Our complex $\operatorname{Sal}(\mathscr{A})$ is essentially the same complex as this defined in [Sa1] but our proof that $\operatorname{Sal}(\mathscr{A})$ has the same homotopy type as $M(\mathscr{A})$ is completely new. In the second subsection we define another simplicial complex $\widehat{S}$ al and prove that there is a natural map $p: \widehat{\mathrm{Sal}} \rightarrow \operatorname{Sal}(\mathscr{A})$ which is the universal cover of $\operatorname{Sal}(\mathscr{A})$ (Theorem 3.7). In particular, $\widehat{\text { Sal }}$ has the same homotopy type as $\widehat{M}$, where $\widehat{M}$ is the universal cover of $M(\mathscr{A})$.

3A. Salvetti's complex. We provide $V$ with an arbitrary scalar product. Let $\mathbb{S}^{l-1}=\{x \in V|| x \mid=1\}$ be the unit sphere. The arrangement $\mathscr{A}$ determines a cellular decomposition of $\mathbb{S}^{l-1}$. With a facet $F \neq\{0\}$ of $\mathscr{A}$ corresponds the (open) cell $\Delta(F)=F \cap \mathbb{S}^{l-1}$, and every open cell of this decomposition of $\mathbb{S}^{l-1}$ has that form.

This cellular decomposition of $\mathbb{S}^{l-1}$ determines a simplicial decomposition of $\mathbb{S}^{l-1}$ (called barycentric subdivision). For every facet $F \neq\{0\}$ of $\mathscr{A}$ we fix a point $x(F) \in \Delta(F)$. A chain $\{0\} \neq F_{0}<F_{1}<\cdots<F_{r}$ of facets of $\mathscr{A}$ determines a simplex $\phi=x\left(F_{0}\right) \vee x\left(F_{1}\right) \vee \cdots \vee x\left(F_{r}\right)$ having $x\left(F_{0}\right), x\left(F_{1}\right), \ldots, x\left(F_{r}\right)$ as vertices, and every simplex of this simplicial decomposition of $\mathbb{S}^{l-1}$ has that form. From now on, we always assume $\mathbb{S}^{l-1}$ to be provided with the simplicial decomposition described above.

Let $\mathbb{B}^{l}=\{x \in V|| x \mid \leq 1\}$ be the unit disc. The simplicial decomposition of $\mathbb{S}^{l-1}$ determines a simplicial decomposition of $\mathbb{B}^{l}$ (called the cone over $\mathbb{S}^{l-1}$ ). We add the vertex $x(\{0\})=0$ to the set of vertices of $\mathbb{S}^{l-1}$. A chain $F_{0}<F_{1}<$ $\cdots<F_{r}$ of facets of $\mathscr{A}$ determines a simplex $\phi=x\left(F_{0}\right) \vee x\left(F_{1}\right) \vee \cdots \vee x\left(F_{r}\right)$ having $x\left(F_{0}\right), x\left(F_{1}\right), \ldots, x\left(F_{r}\right)$ as vertices (recall that $\{0\}$ is a facet of $\mathscr{A}$ ), and every simplex of this simplicial decomposition of $\mathbb{B}^{l}$ has that form. Note that, if $F_{0} \neq\{0\}$, then $\phi=x\left(F_{0}\right) \vee x\left(F_{1}\right) \vee \cdots \vee x\left(F_{r}\right) \subseteq \mathbb{S}^{l-1}$. From now on, we always assume $\mathbb{B}^{l}$ to be provided with the simplicial decomposition described above.

Now we are going to define the (abstract) simplicial complex $\operatorname{Sal}(\mathscr{A})$. For every $X \in \mathscr{L}(\mathscr{A})$ and every chamber $D$ of $\mathscr{A}_{X}$, we fix a point $y(D) \in D$. For every facet $F$ of $\mathscr{A}$ and every chamber $C$, we set

$$
z(F, C)=x(F)+i y\left(C_{F}\right)
$$

(recall that $C_{F}$ is the unique chamber of $\mathscr{A}_{|F|}$ containing $C$ ). Note that $z(F, C) \in\left(F+i C_{F}\right) \subseteq M(C)$. We have $z\left(F_{1}, C_{1}\right)=z\left(F_{2}, C_{2}\right)$ if and only if $F_{1}=F_{2}$ and $\left(C_{1}\right)_{F_{1}}=\left(C_{2}\right)_{F_{2}}$.

Let $V(\mathrm{Sal})$ be an abstract set in bijection with the set of points of $M(\mathscr{A})$ having the form $z(F, C)$ with $F \in \mathscr{F}(\mathscr{A})$ and $C$ a chamber of $\mathscr{A}$. We denote by $\omega(F, C)$ the element of $V(\mathrm{Sal})$ corresponding with $z(F, C)$. We have $\omega\left(F_{1}, C_{1}\right)=\omega\left(F_{2}, C_{2}\right)$ if and only if $F_{1}=F_{2}$ and $\left(C_{1}\right)_{F_{1}}=\left(C_{2}\right)_{F_{2}}$. The set $V(\mathrm{Sal})$ will be the set of vertices of $\operatorname{Sal}(\mathscr{A})$.

Let $F_{1}$ and $F_{2}$ be two facets of $\mathscr{A}$ and let $C$ be a chamber. We set $\omega\left(F_{1}, C\right)<\omega\left(F_{2}, C\right)$ if $F_{1}<F_{2}$. 
Lemma 3.1. The relation " $<$ " is a partial order in $V(\mathrm{Sal})$.

Proof. Pick $\omega_{1}, \omega_{2}, \omega_{3} \in V($ Sal $)$ such that $\omega_{1}<\omega_{2}$ and $\omega_{2}<\omega_{3}$, and let us prove that $\omega_{1}<\omega_{3}$.

Since $\omega_{1}<\omega_{2}$, there exist two facets $F_{1}$ and $F_{2}$ of $\mathscr{A}$ and a chamber $C$ such that $F_{1}<F_{2}, \omega_{1}=\omega\left(F_{1}, C\right)$, and $\omega_{2}=\omega\left(F_{2}, C\right)$. Since $\omega_{2}<$ $\omega_{3}$, there exist two facets $F_{2}^{\prime}$ and $F_{3}$ of $\mathscr{A}$ and a chamber $D$ such that $F_{2}^{\prime}<F_{3}, \omega_{2}=\omega\left(F_{2}^{\prime}, D\right)$, and $\omega_{3}=\omega\left(F_{3}, D\right)$. We have $\omega_{2}=\omega\left(F_{2}, C\right)=$ $\omega\left(F_{2}^{\prime}, D\right)$, thus $F_{2}=F_{2}^{\prime}$ and $C_{F_{2}}=D_{F_{2}}$. The inequality $F_{3}>F_{2}^{\prime}=F_{2}$ implies $\mathscr{A}_{\left|F_{3}\right|} \subseteq \mathscr{A}_{\left|F_{2}\right|}$, thus $C_{F_{3}}=D_{F_{3}}$ (since $C_{F_{2}}=D_{F_{2}}$ ), therefore $\omega_{3}=$ $\omega\left(F_{3}, D\right)=\omega\left(F_{3}, C\right)$. It follows that $\omega_{1}=\omega\left(F_{1}, C\right)<\omega_{3}=\omega\left(F_{3}, C\right)$ (since $\left.F_{1}<F_{2}=F_{2}^{\prime}<F_{3}\right)$.

An $r$-simplex $\Phi$ of $\operatorname{Sal}(\mathscr{A})$ is a $(r+1)$ chain $\omega_{0}<\omega_{1}<\cdots<\omega_{r}$ in $V(\mathrm{Sal})$. We write $\Phi=\omega_{0} \vee \omega_{1} \vee \cdots \vee \omega_{r}$. A subset of a chain is clearly still a chain, so $\operatorname{Sal}(\mathscr{A})$ is well defined.

Let $\phi=x\left(F_{0}\right) \vee \cdots \vee x\left(F_{r}\right)$ be a simplex of $\mathbb{B}^{l}$ and let $C$ be a chamber of $\mathscr{A}$. Then $\phi$ and $C$ determine a simplex $\Phi(\phi, C)$ of $\operatorname{Sal}(\mathscr{A})$ defined by

$$
\Phi(\phi, C)=\omega\left(F_{0}, C\right) \vee \cdots \vee \omega\left(F_{r}, C\right) .
$$

Lemma 3.2. Let $\Phi$ be a simplex of $\operatorname{Sal}(\mathscr{A})$. Then there exist a simplex $\phi$ of $\mathbb{B}^{l}$ and a chamber $C$ of $\mathscr{A}$ such that $\Phi=\Phi(\phi, C)$.

Proof. Write $\Phi=\omega_{0} \vee \omega_{1} \vee \cdots \vee \omega_{r}$, with $\omega_{0}<\omega_{1}<\cdots<\omega_{r}$. Fix a facet $F_{0}$ of $\mathscr{A}$ and a chamber $C$ such that $\omega_{0}=\omega\left(F_{0}, C\right)$. Let us prove, by induction on $i=1, \ldots, r$, that there exists a facet $F_{i}$ of $\mathscr{A}$ such that $\omega_{i}=\omega\left(F_{i}, C\right)$ and $F_{i}>F_{i-1}$. It follows that $\Phi=\Phi(\phi, C)$, where $\phi=x\left(F_{0}\right) \vee x\left(F_{1}\right) \vee \cdots \vee x\left(F_{r}\right)$.

Assume there exists a facet $F_{i-1}$ of $\mathscr{A}$ such that $\omega_{i-1}=\omega\left(F_{i-1}, C\right)$. Since $\omega_{i-1}<\omega_{i}$, there exist two facets $F_{i-1}^{\prime}$ and $F_{i}$ of $\mathscr{A}$ and a chamber $D$ such that $F_{i-1}^{\prime}<F_{i}, \omega_{i-1}=\omega\left(F_{i-1}^{\prime}, D\right)$ and $\omega_{i}=\omega\left(F_{i}, D\right)$. We have $\omega_{i-1}=$ $\omega\left(F_{i-1}, C\right)=\omega\left(F_{i-1}^{\prime}, D\right)$, thus $F_{i-1}=F_{i-1}^{\prime}$ and $C_{F_{i-1}}=D_{F_{i-1}}$. The inequality $F_{i}>F_{i-1}^{\prime}=F_{i-1}$ implies $A_{\left|F_{i}\right|} \subseteq \mathscr{A}_{\left|F_{i-1}\right|}$, thus $C_{F_{i}}=D_{F_{i}}$ (since $C_{F_{i-1}}=D_{F_{i-1}}$ ). It follows that $\omega_{i}=\omega\left(F_{i}, D\right)=\omega\left(F_{i}, C\right)$.

Note that, if $\Phi\left(\phi_{1}, C_{1}\right)=\Phi\left(\phi_{2}, C_{2}\right)$, then $\phi_{1}=\phi_{2}$. The map $\pi: \operatorname{Sal}(\mathscr{A}) \rightarrow$ $\mathbb{B}^{l}$ which sends $\Phi(\phi, C)$ onto $\phi$, for every simplex $\phi$ of $\mathbb{B}^{l}$ and every chamber $C$ of $\mathscr{A}$, is clearly a well-defined simplicial map, and sends every simplex of $\operatorname{Sal}(\mathscr{A})$ onto a simplex of $\mathbb{B}^{l}$ having the same dimension.

For a chamber $C$ of $\mathscr{A}$, we denote by $\mathbb{B}^{l}(C)$ the subcomplex of $\operatorname{Sal}(\mathscr{A})$ generated by the vertices of $\operatorname{Sal}(\mathscr{A})$ having the form $\omega(F, C)$ with $F \in$ $\mathscr{F}(\mathscr{A})$. We have

$$
\mathbb{B}^{l}(C)=\bigcup_{\phi} \Phi(\phi, C),
$$

where the union is over all the simplexes $\phi$ of $\mathbb{B}^{l}$. The restriction $\pi_{C}: \mathbb{B}^{l}(C) \rightarrow$ $\mathbb{B}^{l}$ of $\pi$ to $\mathbb{B}^{l}(C)$ is clearly an isomorphism of simplicial complexes. Moreover,

$$
\operatorname{Sal}(\mathscr{A})=\bigcup_{C} \mathbb{B}^{l}(C),
$$

where the union is over all chambers $C$ of $\mathscr{A}$. 
Theorem 3.3. $\mathrm{Sal}(\mathscr{A})$ has the same homotopy type as $M(\mathscr{A})$.

Proof. With every vertex $\omega$ of $\operatorname{Sal}(\mathscr{A})$ we will associate an open convex subset $U(\omega)$ of $M(\mathscr{A})$. We will prove that $\mathscr{U}=\{U(\omega) \mid \omega \in V($ Sal $)\}$ is a covering of $M(\mathscr{A})$ having $\operatorname{Sal}(\mathscr{A})$ as nerve. Since $U(\omega)$ will be convex for every vertex $\omega$ of $\operatorname{Sal}(\mathscr{A})$, any nonempty intersection of elements of $\mathscr{U}$ will be convex (thus contractible). This implies, by [We], that $\operatorname{Sal}(\mathscr{A})$ has the same homotopy type as $M(\mathscr{A})$.

For a simplex $\phi$ of $\mathbb{S}^{l-1}$, we write

$$
K(\phi)=\{\lambda x \mid x \in \phi \text { and } \lambda>0\} .
$$

Note that, if $\phi=x\left(F_{0}\right) \vee \cdots \vee x\left(F_{r}\right)$ with $\{0\} \neq F_{0}<F_{1}<\cdots<F_{r}$, then $K(\phi) \subseteq F_{r}$. Furthermore, the family $\left\{K(\phi) \mid \phi\right.$ a simplex of $\left.\mathbb{S}^{l-1}\right\}$ is a partition of $V-\{0\}$.

Let $F$ be a facet of $\mathscr{A}$ and let $C$ be a chamber. If $F=\{0\}$, then we set

$$
U(\omega(F, C))=(V+i C) .
$$

If $F \neq\{0\}$, then we set

$$
U(\omega(F, C))=\left(\bigcup_{\phi} K(\phi)\right)+i C_{F},
$$

where the union is over all the simplexes $\phi$ of $\mathbb{S}^{l-1}$ having $x(F)$ as vertex. We obviously have $U\left(\omega\left(F_{1}, C_{1}\right)\right)=U\left(\omega\left(F_{2}, C_{2}\right)\right)$ if $\omega\left(F_{1}, C_{1}\right)=\omega\left(F_{2}, C_{2}\right)$, thus $U(\omega)$ is well defined for every vertex $\omega$ of $\operatorname{Sal}(\mathscr{A})$. Moreover, $U(\omega)$ is clearly an open convex subset of $V_{\mathbb{C}}$.

Now, we are going to prove successively the following four assertions.

(1) $U(\omega) \subseteq M(\mathscr{A})$ for every vertex $\omega$ of $\operatorname{Sal}(\mathscr{A})$.

(2) $M(\mathscr{A}) \subseteq \bigcup_{\omega} U(\omega)$, where the union is over all the vertices $\omega$ of $\operatorname{Sal}(\mathscr{A})$.

(3) Let $\omega_{0}, \omega_{1}, \ldots, \omega_{r}$ be $(r+1)$ distinct vertices of $\operatorname{Sal}(\mathscr{A})$. If $U\left(\omega_{0}\right) \cap$ $U\left(\omega_{1}\right) \cap \cdots \cap U\left(\omega_{r}\right) \neq \varnothing$, then $\omega_{0}, \omega_{1}, \ldots, \omega_{r}$ are the vertices of a simplex $\Phi$ of $\operatorname{Sal}(\mathscr{A})$.

(4) If $\omega_{0}, \omega_{1}, \ldots, \omega_{r}$ are the vertices of a simplex $\Phi$ of $\operatorname{Sal}(\mathscr{A})$, then $U\left(\omega_{0}\right) \cap U\left(\omega_{1}\right) \cap \cdots \cap U\left(\omega_{r}\right) \neq \varnothing$.

Assertions (1)-(4) obviously prove that $\mathscr{U}=\{U(\omega) \mid \omega \in V(\mathrm{Sal})\}$ is a covering of $M(\mathscr{A})$ having $\operatorname{Sal}(\mathscr{A})$ as nerve.

(1) Let $F$ be a facet of $\mathscr{A}$ and let $C$ be a chamber. If $F=\{0\}$, then $U(\omega(F, C))=(V+i C)$ is obviously included in $M(\mathscr{A})$. Now, assume $F \neq$ $\{0\}$. Pick $z=(x+i y) \in U(\omega(F, C))$. There is a simplex $\phi$ of $\mathbb{S}^{l-1}$ having $x(F)$ as vertex and such that $x \in K(\phi)$. We write $\phi=x\left(F_{0}\right) \vee x\left(F_{1}\right) \vee \cdots \vee x\left(F_{r}\right)$, with $\{0\} \neq F_{0}<F_{1}<\cdots<F_{r}$. We have

$$
K(\phi) \subseteq F_{r} \Rightarrow x \in F_{r},
$$

and

$$
F \leq F_{r} \Rightarrow C_{F} \subseteq C_{F_{r}} \Rightarrow y \in C_{F_{r}} .
$$

Therefore $z=(x+i y) \in\left(F_{r}+i C_{F_{r}}\right) \subseteq M(\mathscr{A})$.

(2) Pick $z=(x+i y) \in M(\mathscr{A})$. If $x=0$, then $x \in H$ for every $H \in \mathscr{A}$, thus $y \notin H$ for every $H \in \mathscr{A}$, thus there exists a chamber $C$ of $\mathscr{A}$ such that $y \in C$. Therefore $z=(x+i y) \in(V+i C)=U(\omega(\{0\}, C))$. 
Now, assume $x \neq 0$. There exists a simplex $\phi$ of $\mathbb{S}^{l-1}$ such that $x \in K(\phi)$. We write $\phi=x\left(F_{0}\right) \vee x\left(F_{1}\right) \vee \cdots \vee x\left(F_{r}\right)$ with $\{0\} \neq F_{0}<F_{1}<\cdots<F_{r}$. Since $x \in K(\phi) \subseteq F_{r}$, there is no hyperplane $H \in \mathscr{A}$ containing $F_{r}$ which contains $y$, thus there is a chamber $D$ of $\mathscr{A}_{\left|F_{r}\right|}$ such that $y \in D$. Pick a chamber $C$ of $\mathscr{A}$ such that $C_{F_{r}}=D$. Then

$$
z=(x+i y) \in\left(K(\phi)+i C_{F_{r}}\right) \subseteq U\left(\omega\left(F_{r}, C\right)\right) .
$$

(3) Let $\omega_{0}, \omega_{1}, \ldots, \omega_{r}$ be $(r+1)$ distinct vertices of $\operatorname{Sal}(\mathscr{A})$ such that $U\left(\omega_{0}\right) \cap U\left(\omega_{1}\right) \cap \cdots \cap U\left(\omega_{r}\right) \neq \varnothing$. Write $\omega_{i}=\omega\left(F_{i}, C_{i}\right)$, where $F_{i} \in \mathscr{F}(\mathscr{A})$ and $C_{i}$ is a chamber of $\mathscr{A}$, for $i=0,1, \ldots, r$. Pick $z=(x+i y) \in \bigcap_{i=0}^{r} U\left(\omega_{i}\right)$.

Case a. Assume $F_{0}=\{0\}$.

Suppose there exists an $i \in\{1, \ldots, r\}$ such that $F_{i}=\{0\}$. Then

$$
\begin{aligned}
z & =(x+i y) \in U\left(\omega_{0}\right) \cap U\left(\omega_{i}\right)=\left(V+i C_{0}\right) \cap\left(V+i C_{i}\right) \\
& \Rightarrow y \in C_{0} \cap C_{i} \\
& \Rightarrow C_{0} \cap C_{i} \neq \varnothing \\
& \Rightarrow C_{0}=C_{i} \\
& \Rightarrow \omega_{0}=\omega\left(\{0\}, C_{0}\right)=\omega_{i}=\omega\left(\{0\}, C_{i}\right) .
\end{aligned}
$$

This contradicts the fact that $\omega_{0} \neq \omega_{i}$. Therefore $F_{i} \neq\{0\}$ for $i=1, \ldots, r$.

There is a simplex $\phi_{i}$ of $\mathbb{S}^{l-1}$ having $x\left(F_{i}\right)$ as vertex and such that $x \in$ $K\left(\phi_{i}\right)$, for $i=1, \ldots, r$. Since $\left\{K(\phi) \mid \phi\right.$ a simplex of $\left.\mathbb{S}^{l-1}\right\}$ is a partition of $V-\{0\}$, we have $\phi_{1}=\cdots=\phi_{r}$. Therefore $x\left(F_{1}\right), \ldots, x\left(F_{r}\right)$ are vertices of a same simplex $\phi$ of $\mathbb{S}^{l-1}$, thus $\left\{F_{1}, \ldots, F_{r}\right\}$ is a chain. Assume $\{0\}=F_{0}<$ $F_{1} \leq \cdots \leq F_{r}$. For $i=1, \ldots, r$ we have

$$
\begin{aligned}
y \in C_{0} \text { and } y \in\left(C_{i}\right)_{F_{i}} & \Rightarrow y \in\left(C_{0}\right)_{F_{i}} \text { and } y \in\left(C_{i}\right)_{F_{i}} \\
& \Rightarrow\left(C_{0}\right)_{F_{i}} \cap\left(C_{i}\right)_{F_{i}} \neq \varnothing \\
& \Rightarrow\left(C_{0}\right)_{F_{i}}=\left(C_{i}\right)_{F_{i}} \\
& \Rightarrow \omega_{i}=\omega\left(F_{i}, C_{i}\right)=\omega\left(F_{i}, C_{0}\right) .
\end{aligned}
$$

Moreover, we have $F_{i-1} \neq F_{i}$ for $i=1, \ldots, r$; otherwise $\omega_{i-1}=\omega\left(F_{i-1}, C_{0}\right)$ $=\omega_{i}=\omega\left(F_{i}, C_{0}\right)$. It follows that $\omega_{0}, \omega_{1}, \ldots, \omega_{r}$ are the vertices of the simplex

of $\operatorname{Sal}(\mathscr{A})$.

$$
\Phi=\omega\left(F_{0}, C_{0}\right) \vee \omega\left(F_{1}, C_{0}\right) \vee \cdots \vee \omega\left(F_{r}, C_{0}\right)
$$

Case b. Assume $F_{i} \neq\{0\}$ for $i=0,1, \ldots, r$.

There is a simplex $\phi_{i}$ of $\mathbb{S}^{l-1}$ having $x\left(F_{i}\right)$ as vertex and such that $x \in$ $K\left(\phi_{i}\right)$, for $i=0,1, \ldots, r$. Since $\left\{K(\phi) \mid \phi\right.$ a simplex of $\left.\mathbb{S}^{l-1}\right\}$ is a partition of $V-\{0\}$, we have $\phi_{0}=\phi_{1}=\cdots=\phi_{r}$. Therefore $\left\{F_{0}, F_{1}, \ldots, F_{r}\right\}$ is a chain. Assume $\{0\} \neq F_{0} \leq F_{1} \leq \cdots \leq F_{r}$. For $i=1, \ldots, r$ we have

$$
y \in\left(C_{0}\right)_{F_{0}}, \quad F_{i} \geq F_{0} \text { and } \begin{aligned}
y \in\left(C_{i}\right)_{F_{i}} & \Rightarrow y \in\left(C_{0}\right)_{F_{i}} \text { and } y \in\left(C_{i}\right)_{F_{i}} \\
& \Rightarrow\left(C_{0}\right)_{F_{i}} \cap\left(C_{i}\right)_{F_{i}} \neq \varnothing \\
& \Rightarrow\left(C_{0}\right)_{F_{i}}=\left(C_{i}\right)_{F_{i}} \\
& \Rightarrow \omega_{i}=\omega\left(F_{i}, C_{i}\right)=\omega\left(F_{i}, C_{0}\right) .
\end{aligned}
$$

Moreover, we have $F_{i-1} \neq F_{i}$ for $i=1, \ldots, r$; otherwise $\omega_{i-1}=\omega\left(F_{i-1}, C_{0}\right)$ $=\omega_{i}=\omega\left(F_{i}, C_{0}\right)$. It follows that $\omega_{0}, \omega_{1}, \ldots, \omega_{r}$ are the vertices of the 
simplex

$$
\Phi=\omega\left(F_{0}, C_{0}\right) \vee \omega\left(F_{1}, C_{0}\right) \vee \cdots \vee \omega\left(F_{r}, C_{0}\right)
$$

of $\operatorname{Sal}(\mathscr{A})$.

(4) Let $\Phi=\Phi(\phi, C)$ be a simplex of $\operatorname{Sal}(\mathscr{A})$. We write $\phi=x\left(F_{0}\right) \vee$ $x\left(F_{1}\right) \vee \cdots \vee x\left(F_{r}\right)$ with $F_{0}<F_{1}<\cdots<F_{r}$. The vertices of $\Phi$ are $\omega\left(F_{0}, C\right)$, $\omega\left(F_{1}, C\right), \ldots, \omega\left(F_{r}, C\right)$. Let us prove that $\bigcap_{i=0}^{r} U\left(\omega\left(F_{i}, C\right)\right)=\varnothing$.

Case a. Assume $F_{0}=\{0\}$.

Consider the simplex $\phi^{\prime}=x\left(F_{1}\right) \vee \cdots \vee x\left(F_{r}\right)$ of $\mathbb{S}^{l-1}$. Pick $x \in K\left(\phi^{\prime}\right)$ and $y \in C$, and write $z=x+i y$. We obviously have $z \in(V+i C)=U\left(\omega\left(F_{0}, C\right)\right)$. The simplex $\phi^{\prime}$ has $x\left(F_{i}\right)$ as vertex, $x \in K\left(\phi^{\prime}\right)$ and $y \in C \subseteq C_{F_{i}}$, thus $z=(x+$ $i y) \in U\left(\omega\left(F_{i}, C\right)\right)$ for $i=1, \ldots, r$. It follows that $z \in \bigcap_{i=0}^{r} U\left(\omega\left(F_{i}, C\right)\right)$.

Case b. Assume $F_{0} \neq\{0\}$.

Then $\phi$ is a simplex of $\mathbb{S}^{l-1}$. Pick $x \in K(\phi)$ and $y \in C$, and write $z=x+i y$. The simplex $\phi$ has $x\left(F_{i}\right)$ as vertex, $x \in K(\phi)$ and $y \in C \subseteq C_{F_{i}}$, thus $z=(x+i y) \in U\left(\omega\left(F_{i}, C\right)\right)$ for $i=0,1, \ldots, r$. It follows that $z \in$ $\bigcap_{i=0}^{r} U\left(\omega\left(F_{i}, C\right)\right)$.

3B. Universal cover of Salvetti's complex. Now, we are going to define the (abstract) simplicial complex $\widehat{\text { Sal }}$.

Throughout this subsection, we denote by $\rho:(\widehat{\Gamma}, \sim) \rightarrow(\Gamma(\mathscr{A}), \sim)$ the universal cover of $(\Gamma(\mathscr{A}), \sim)$, and by $q: \widehat{M} \rightarrow M(\mathscr{A})$ the universal cover of $M(\mathscr{A})$, as defined in $\S 2$.

Let $C$ be a chamber of $\mathscr{A}$. For every facet $F \in \mathscr{F}(\mathscr{A})$ we have

$$
z(F, C)=\left(x(F)+i y\left(C_{F}\right)\right) \in\left(F+i C_{F}\right) \subseteq M(C) .
$$

By Lemma 2.7,

$$
q^{-1}(M(C))=\bigcup_{v \in \rho^{-1}(C)} \widehat{M}(v),
$$

and this union is disjoint. Moreover, recall that $q$ sends $\widehat{M}(v)$ homeomorphically onto $M(v)=M(C)$ for every vertex $v \in \rho^{-1}(C)$. This implies that, for every facet $F \in \mathscr{F}(\mathscr{A})$ and every vertex $v \in V(\widehat{\Gamma})$, there exists a unique point $e(F, v) \in \widehat{M}(v)$ such that $q(e(F, v))=z(F, \rho(v))$ (i.e., $e(F, v)$ is the lift of $z(F, C)$ into $\widehat{M}(v)$, where $C=\rho(v))$.

Let $V(\widehat{\text { Sal }})$ be an abstract set in bijection with the set of points of $\widehat{M}$ having the form $e(F, v)$ with $F \in \mathscr{F}(\mathscr{A})$ and $v \in V(\widehat{\Gamma})$. We denote by $\hat{\omega}(F, v)$ the element of $V(\widehat{\mathrm{S} a l})$ corresponding to $e(F, v)$. The set $V(\widehat{\mathrm{S}} \mathrm{al})$ will be the set of vertices of $\widehat{\mathrm{S} a l}$.

Lemma 3.4. Let $F_{1}, F_{2}$ be two facets of $\mathscr{A}$, and let $v_{1}, v_{2}$ be two vertices of $\widehat{\Gamma}$. Then

$$
\hat{\omega}\left(F_{1}, v_{1}\right)=\hat{\omega}\left(F_{2}, v_{2}\right)
$$

if and only if $F_{1}=F_{2} \subseteq Z\left(v_{1}, v_{2}\right)$ and $\rho\left(v_{1}\right)_{F_{1}}=\rho\left(v_{2}\right)_{F_{2}}$.

Proof. Assume $\hat{\omega}\left(F_{1}, v_{1}\right)=\hat{\omega}\left(F_{2}, v_{2}\right)$ (thus $\left.e\left(F_{1}, v_{1}\right)=e\left(F_{2}, v_{2}\right)\right)$. Since $e\left(F_{1}, v_{1}\right) \in \widehat{M}\left(v_{1}\right)$ and $e\left(F_{2}, v_{2}\right) \in \widehat{M}\left(v_{2}\right)$, by Lemma 2.6 ,

$$
\begin{aligned}
q\left(e\left(F_{1}, v_{1}\right)\right) & =z\left(F_{1}, \rho\left(v_{1}\right)\right)=q\left(e\left(F_{2}, v_{2}\right)\right) \\
& =z\left(F_{2}, \rho\left(v_{2}\right)\right) \in M\left(v_{1}\right) \cap M\left(v_{2}\right) \cap\left(Z\left(v_{1}, v_{2}\right)+i V\right) .
\end{aligned}
$$


The equality $z\left(F_{1}, \rho\left(v_{1}\right)\right)=z\left(F_{2}, \rho\left(v_{2}\right)\right)$ implies $F_{1}=F_{2}$ and $\rho\left(v_{1}\right)_{F_{1}}=$ $\rho\left(v_{2}\right)_{F_{2}}$. On the other hand, $x\left(F_{1}\right) \in Z\left(v_{1}, v_{2}\right)$, the set $Z\left(v_{1}, v_{2}\right)$ is a union of facets of $\mathscr{A}$ and $x\left(F_{1}\right) \in F_{1}$, thus $F_{1} \subseteq Z\left(v_{1}, v_{2}\right)$.

Now, assume $F_{1}=F_{2} \subseteq Z\left(v_{1}, v_{2}\right)$ and $\rho\left(v_{1}\right)_{F_{1}}=\rho\left(v_{2}\right)_{F_{2}}$. This implies

$$
\begin{gathered}
z\left(F_{1}, \rho\left(v_{1}\right)\right)=z\left(F_{2}, \rho\left(v_{2}\right)\right) \in\left(F_{1}+i \rho\left(v_{1}\right)_{F_{1}}\right) \cap\left(F_{2}+i \rho\left(v_{2}\right)_{F_{2}}\right) \\
\cap\left(Z\left(v_{1}, v_{2}\right)+i V\right) \subseteq M\left(v_{1}\right) \cap M\left(v_{2}\right) \cap\left(Z\left(v_{1}, v_{2}\right)+i V\right) .
\end{gathered}
$$

It follows, by Lemma 2.6, that

$$
z\left(F_{1}, \rho\left(v_{1}\right)\right)=z\left(F_{2}, \rho\left(v_{2}\right)\right) \in q\left(\widehat{M}\left(v_{1}\right) \cap \widehat{M}\left(v_{2}\right)\right) .
$$

The map $q$ sends $\widehat{M}\left(v_{1}\right)$ homeomorphically onto $M\left(v_{1}\right)$, the point $e\left(F_{1}, v_{1}\right)$ is the lift of $z\left(F_{1}, \rho\left(v_{1}\right)\right)$ into $\widehat{M}\left(v_{1}\right)$, and $z\left(F_{1}, \rho\left(v_{1}\right)\right) \in q\left(\widehat{M}\left(v_{1}\right) \cap \widehat{M}\left(v_{2}\right)\right)$, thus $e\left(F_{1}, v_{1}\right) \in \widehat{M}\left(v_{1}\right) \cap \widehat{M}\left(v_{2}\right)$. It follows that $e\left(F_{1}, v_{1}\right)$ is the unique lift of $z\left(F_{2}, \rho\left(v_{2}\right)\right)=z\left(F_{1}, \rho\left(v_{1}\right)\right)$ into $\widehat{M}\left(v_{2}\right)$, thus $e\left(F_{1}, v_{1}\right)=e\left(F_{2}, v_{2}\right)$, therefore $\hat{\omega}\left(F_{1}, v_{1}\right)=\hat{\omega}\left(F_{2}, v_{2}\right)$.

Let $F_{1}$ and $F_{2}$ be two facets of $\mathscr{A}$ and let $v$ be a vertex of $\widehat{\Gamma}$. We set $\hat{\omega}\left(F_{1}, v\right)<\hat{\omega}\left(F_{2}, v\right)$ if $F_{1}<F_{2}$.

Lemma 3.5. The relation " $<$ " is a partial order in $V(\widehat{\text { Sal }})$.

Proof. Pick $\hat{\omega}_{1}, \hat{\omega}_{2}, \hat{\omega}_{3} \in V(\widehat{\text { Sal }})$ such that $\hat{\omega}_{1}<\hat{\omega}_{2}$ and $\hat{\omega}_{2}<\hat{\omega}_{3}$, and let us prove that $\hat{\omega}_{1}<\hat{\omega}_{3}$.

Since $\hat{\omega}_{1}<\hat{\omega}_{2}$, there exist two facets $F_{1}$ and $F_{2}$ of $\mathscr{A}$ and a vertex $v$ of $\widehat{\Gamma}$ such that $F_{1}<F_{2}, \hat{\omega}_{1}=\hat{\omega}\left(F_{1}, v\right)$, and $\hat{\omega}_{2}=\hat{\omega}\left(F_{2}, v\right)$. Since $\hat{\omega}_{2}<\hat{\omega}_{3}$, there exist two facets $F_{2}^{\prime}$ and $F_{3}$ of $\mathscr{A}$ and a vertex $w$ of $\widehat{\Gamma}$ such that $F_{2}^{\prime}<F_{3}$, $\hat{\omega}_{2}=\hat{\omega}\left(F_{2}^{\prime}, w\right)$, and $\hat{\omega}_{3}=\hat{\omega}\left(F_{3}, w\right)$. We have $\hat{\omega}_{2}=\hat{\omega}\left(F_{2}, v\right)=\hat{\omega}\left(F_{2}^{\prime}, w\right)$, thus, by Lemma 3.4, $F_{2}=F_{2}^{\prime} \subseteq Z(v, w)$ and $\rho(v)_{F_{2}}=\rho(w)_{F_{2}}$. Note that $Z(v, w)$ is a union of facets of $\mathscr{A}$ and is an open subset of $V$, thus, if $F$ and $G$ are two facets of $\mathscr{A}$ such that $G \geq F$ and $F \subseteq Z(v, w)$, then $G \subseteq$ $Z(v, w)$. Since $F_{3}>F_{2}^{\prime}=F_{2}$ and $F_{2} \subseteq Z(v, w)$, we have $F_{3} \subseteq Z(v, w)$. Furthermore, $\mathscr{A}_{\left|F_{2}\right|} \supseteq \mathscr{A}_{\left|F_{3}\right|}$ and $\rho(v)_{F_{2}}=\rho(w)_{F_{2}}$, thus $\rho(v)_{F_{3}}=\rho(w)_{F_{3}}$. It follows, by Lemma 3.4, that $\hat{\omega}_{3}=\hat{\omega}\left(F_{3}, w\right)=\hat{\omega}\left(F_{3}, v\right)$. Therefore $\hat{\omega}_{1}=$ $\hat{\omega}\left(F_{1}, v\right)<\hat{\omega}_{3}=\hat{\omega}\left(F_{3}, v\right)$ (since $\left.F_{1}<F_{2}=F_{2}^{\prime}<F_{3}\right)$.

An $r$-simplex $\Phi$ of $\widehat{\text { Sal }}$ is a $(r+1)$ chain $\hat{\omega}_{0}<\hat{\omega}_{1}<\cdots<\hat{\omega}_{r}$ in $V(\widehat{\text { Sal }})$. We write $\Phi=\hat{\omega}_{0} \vee \hat{\omega}_{1} \vee \cdots \vee \hat{\omega}_{r}$. A subset of a chain is clearly still a chain, so

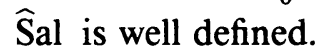

Let $\phi=x\left(F_{0}\right) \vee x\left(F_{1}\right) \vee \cdots \vee x\left(F_{r}\right)$ be a simplex of $\mathbb{B}^{l}$ and let $v$ be a vertex of $\widehat{\Gamma}$. Then $\phi$ and $v$ determine a simplex $\Phi(\phi, v)$ of $\widehat{\text { Sal defined by }}$

$$
\Phi(\phi, v)=\hat{\omega}\left(F_{0}, v\right) \vee \hat{\omega}\left(F_{1}, v\right) \vee \cdots \vee \hat{\omega}\left(F_{r}, v\right) .
$$

Lemma 3.6. Let $\Phi$ be a simplex of $\widehat{\text { Sal }}$. Then there exist a simplex $\phi$ of $\mathbb{B}^{l}$ and a vertex $v$ of $\widehat{\Gamma}$ such that $\Phi=\Phi(\phi, v)$.

Proof. Write $\Phi=\hat{\omega}_{0} \vee \hat{\omega}_{1} \vee \cdots \vee \hat{\omega}_{r}$ with $\hat{\omega}_{0}<\hat{\omega}_{1}<\cdots<\hat{\omega}_{r}$. Fix a facet $F_{0}$ of $\mathscr{A}$ and a vertex $v$ of $\widehat{\Gamma}$ such that $\hat{\omega}_{0}=\hat{\omega}\left(F_{0}, v\right)$. Let us prove, by induction on $i=1, \ldots, r$, that there exists a facet $F_{i}$ of $\mathscr{A}$ such that $\hat{\omega}_{i}=\hat{\omega}\left(F_{i}, v\right)$ and $F_{i}>F_{i-1}$. It follows that $\Phi=\Phi(\phi, v)$, where $\phi=x\left(F_{0}\right) \vee x\left(F_{1}\right) \vee \cdots \vee x\left(F_{r}\right)$. 
Assume there exists a facet $F_{i-1}$ of $\mathscr{A}$ such that $\hat{\omega}_{i-1}=\hat{\omega}\left(F_{i-1}, v\right)$. Since $\hat{\omega}_{i-1}<\hat{\omega}_{i}$, there exist two facets $F_{i-1}^{\prime}$ and $F_{i}$ of $\mathscr{A}$ and a vertex $w$ of $\widehat{\Gamma}$ such that $F_{i-1}^{\prime}<F_{i}, \hat{\omega}_{i-1}=\hat{\omega}\left(F_{i-1}^{\prime}, w\right)$ and $\hat{\omega}_{i}=\hat{\omega}\left(F_{i}, w\right)$. We have $\hat{\omega}_{i-1}=$ $\hat{\omega}\left(F_{i-1}, v\right)=\hat{\omega}\left(F_{i-1}^{\prime}, w\right)$, thus, by Lemma 3.4, $F_{i-1}=F_{i-1}^{\prime} \subseteq Z(v, w)$ and $\rho(v)_{F_{i-1}}=\rho(w)_{F_{i-1}}$. Since $F_{i}>F_{i-1}^{\prime}=F_{i-1}$ and $F_{i-1} \subseteq Z(v, w)$, we have $F_{i} \subseteq Z(v, w)$. Since $F_{i}>F_{i-1}$ and $\rho(v)_{F_{i-1}}=\rho(w)_{F_{i-1}}$, we have $\rho(v)_{F_{i}}=$ $\rho(w)_{F_{i}}$. It follows, by Lemma 3.4, that $\hat{\omega}_{i}=\hat{\omega}\left(F_{i}, w\right)=\hat{\omega}\left(F_{i}, v\right)$.

Note that, if $\Phi\left(\phi_{1}, v_{1}\right)=\Phi\left(\phi_{2}, v_{2}\right)$, then $\phi_{1}=\phi_{2}$. The map $\hat{\pi}: \widehat{\text { Sal }} \rightarrow \mathbb{B}^{l}$ which sends $\Phi(\phi, v)$ onto $\phi$, for every simplex $\phi$ of $\mathbb{B}^{l}$ and every vertex $v$ of $\widehat{\Gamma}$, is clearly a well-defined simplicial map and sends every simplex of $\widehat{S}$ al onto a simplex of $\mathbb{B}^{l}$ having the same dimension.

For a vertex $v$ of $\widehat{\Gamma}$, we denote by $\mathbb{B}^{l}(v)$ the subcomplex of $\widehat{\text { Sal generated }}$ by the vertices of $\widehat{S}$ al having the form $\hat{\omega}(F, v)$ with $F \in \mathscr{F}(\mathscr{A})$. We have

$$
\mathbf{B}^{l}(v)=\bigcup_{\phi} \Phi(\phi, v)
$$

where the union is over all the simplexes $\phi$ of $\mathbb{B}^{l}$. The restriction $\hat{\pi}_{v}: \mathbb{B}^{l}(v) \rightarrow$ $\mathbb{B}^{l}$ of $\hat{\pi}$ to $\mathbb{B}^{l}(v)$ is clearly an isomorphism of simplicial complexes. Moreover,

$$
\widehat{\mathrm{Sal}}=\bigcup_{v \in V(\widehat{\Gamma})} \mathbb{B}^{l}(v)
$$

Consider the map $p: \widehat{\operatorname{Sal}} \rightarrow \operatorname{Sal}(\mathscr{A})$ which sends $\Phi(\phi, v)$ onto $\Phi(\phi, \rho(v))$ for every simplex $\phi$ of $\mathbb{B}^{l}$ and every vertex $v$ of $\widehat{\Gamma}$. It is clearly a simplicial

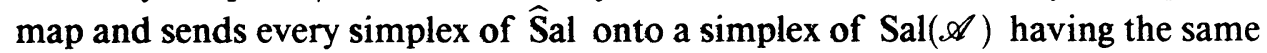
dimension.

Theorem 3.7. The map $p: \widehat{\mathrm{Sal}} \rightarrow \operatorname{Sal}(\mathscr{A})$ is the universal cover of $\mathrm{Sal}(\mathscr{A})$. In particular, $\widehat{\text { Sal }}$ has the same homotopy type as $\widehat{M}$.

Remark. Using the same ideas as in the proof of Theorem 3.3, one can show

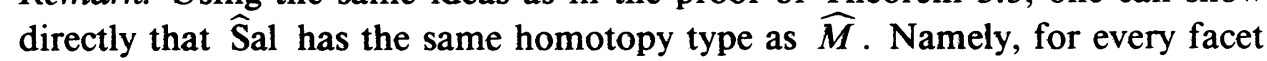
$F$ of $\mathscr{A}$ and every chamber $C$, we have $U(\omega(F, C)) \subseteq M(C)$. Thus, if $F \in \mathscr{F}(\mathscr{A})$ and $v \in V(\widehat{\Gamma})$, then there exists a unique open "convex" set $U(\hat{\omega}(F, v)) \subseteq \widehat{M}(v)$ such that $q(U(\hat{\omega}(F, v)))=U(\omega(F, \rho(v)))$. One can show that $U\left(\hat{\omega}\left(F_{1}, v_{1}\right)\right)=U\left(\hat{\omega}\left(F_{2}, v_{2}\right)\right)$ if $\hat{\omega}\left(F_{1}, v_{1}\right)=\hat{\omega}\left(F_{2}, v_{2}\right)$, the set $\mathscr{U}=\{U(\hat{\omega}) \mid \hat{\omega} \in V(\widehat{\mathbf{S}} \mathrm{al})\}$ is a covering of $\widehat{M}$ having $\widehat{\text { Sal }}$ as nerve, and every nonempty intersection of elements of $\mathscr{U}$ is "convex". Nevertheless, we present here a different proof which may be more complicated but which carries more information; for example, we will give explicitly a homotopy equivalence between $\widehat{\text { Sal }}$ and $\widehat{M}$.

The following Lemma 3.8 is a preliminary result to the proof of Theorem 3.7.

For $(r+1)$ distinct points $p_{0}, p_{1}, \ldots, p_{r}$ of a real vector space, we set

$$
\Delta\left(p_{0}, p_{1}, \ldots, p_{r}\right)=\left\{\sum_{i=0}^{r} t_{i} p_{i} \mid 0<t_{i} \leq 1 \text { and } \sum_{i=0}^{r} t_{i}=1\right\} \text {. }
$$


Consider the map $l: \operatorname{Sal}(\mathscr{A}) \rightarrow V_{\mathbb{C}}$ which sends a simplex

$$
\Phi=\omega\left(F_{0}, C\right) \vee \omega\left(F_{1}, C\right) \vee \cdots \vee \omega\left(F_{r}, C\right)
$$

of $\operatorname{Sal}(\mathscr{A})$ onto $\Delta\left(z\left(F_{0}, C\right), z\left(F_{1}, C\right), \ldots, z\left(F_{r}, C\right)\right)$. This maps sends $\omega(F, C)$ onto $z(F, C)$ for every facet $F$ of $\mathscr{A}$ and every chamber $C$. It is obviously well defined.

Lemma 3.8. (i) Let $\phi$ be a simplex of $\mathbb{B}^{l}$, and let $C$ be a chamber of $\mathscr{A}$. Write $\phi=x\left(F_{0}\right) \vee x\left(F_{1}\right) \vee \cdots \vee x\left(F_{r}\right)$ with $F_{0}<F_{1}<\cdots<F_{r}$. Then $l(\Phi(\phi, C)) \subseteq$ $\left(F_{r}+i C_{F_{r}}\right)$. In particular, $l(\operatorname{Sal}(\mathscr{A})) \subseteq M(\mathscr{A})$.

(ii) The map $l$ is injective.

(iii) The map $l: \operatorname{Sal}(\mathscr{A}) \rightarrow M(\mathscr{A})$ is a homotopy equivalence.

Proof. (i) Let $\phi$ be a simplex of $\mathbb{B}^{l}$, and let $C$ be a chamber of $\mathscr{A}$. Write $\phi=x\left(F_{0}\right) \vee x\left(F_{1}\right) \vee \cdots \vee x\left(F_{r}\right)$ with $F_{0}<F_{1}<\cdots<F_{r}$. Pick $z=(x+i y)=$ $\sum_{i=0}^{r} \lambda_{i} z\left(F_{i}, C\right) \in l(\Phi(\phi, C))$. We have $\Delta\left(x\left(F_{0}\right), x\left(F_{1}\right), \ldots, x\left(F_{r}\right)\right) \subseteq F_{r}$, thus $x=\sum_{i=0}^{r} \lambda_{i} x\left(F_{i}\right) \in F_{r}$. On the other hand, $y\left(C_{F_{i}}\right) \in C_{F_{i}} \subseteq C_{F_{r}}$ for $i=0,1, \ldots, r$, thus $y=\sum_{i=0}^{r} \lambda_{i} y\left(C_{F_{i}}\right) \in C_{F_{r}}$ (since $C_{F_{r}}$ is convex).

(ii) Let $\phi$ and $\psi$ be two simplexes of $\mathbb{B}^{l}$, and let $C$ and $D$ be two chambers of $\mathscr{A}$. Write $\phi=x\left(F_{0}\right) \vee x\left(F_{1}\right) \vee \cdots \vee x\left(F_{r}\right)$ with $F_{0}<F_{1}<\cdots<F_{r}$, and $\psi=$ $x\left(G_{0}\right) \vee x\left(G_{1}\right) \vee \cdots \vee x\left(G_{s}\right)$ with $G_{0}<G_{1}<\cdots<G_{s}$. Let us prove, by induction on $r$, that, if $l(\Phi(\phi, C)) \cap l(\Phi(\psi, D)) \neq \varnothing$, then $\Phi(\phi, C)=\Phi(\psi, D)$.

Pick $z=(x+i y) \in \imath(\Phi(\phi, C)) \cap \imath(\Phi(\psi, D))$. Write $z=\sum_{i=0}^{r} t_{i} z\left(F_{i}, C\right)=$ $\sum_{j=0}^{s} t_{j}^{\prime} z\left(G_{j}, D\right)$, where $0<t_{i}, t_{j}^{\prime} \leq 1$ and $\sum_{i=0}^{r} t_{i}=\sum_{j=0}^{s} t_{j}^{\prime}=1$. Recall that $\{x(F) \mid F \in \mathscr{F}(\mathscr{A})\}$ is the set of vertices of a triangulation of $\mathbb{B}^{l}$. Since $x=$ $\sum_{i=0}^{r} t_{i} x\left(F_{i}\right)=\sum_{j=0}^{s} t_{j}^{\prime} x\left(G_{j}\right)$, we have $r=s, F_{i}=G_{i}$ (for $\left.i=0,1, \ldots, r\right)$ and $t_{i}=t_{i}^{\prime}$ (for $\left.i=0, \ldots, r\right)$. In particular, $\phi=\psi$. Moreover,

$$
\varnothing \neq l(\Phi(\phi, C)) \cap l(\Phi(\phi, D)) \subseteq\left(F_{r}+i C_{F_{r}}\right) \cap\left(F_{r}+i D_{F_{r}}\right),
$$

thus $C_{F_{r}} \cap D_{F_{r}} \neq \varnothing$, therefore $C_{F_{r}}=D_{F_{r}}$. It follows that

$$
\begin{aligned}
z^{\prime} & =\left(\sum_{i=0}^{r-1} t_{i}\right)^{-1}\left(z-t_{r} z\left(F_{r}, C\right)\right) \\
& =\left(\sum_{i=0}^{r-1} t_{i}\right)^{-1}\left(z-t_{r} z\left(F_{r}, D\right)\right) \in l\left(\Phi\left(\phi^{\prime}, C\right)\right) \cap l\left(\Phi\left(\phi^{\prime}, D\right)\right),
\end{aligned}
$$

where $\phi^{\prime}=x\left(F_{0}\right) \vee x\left(F_{1}\right) \vee \cdots \vee x\left(F_{r-1}\right)$. By the inductive hypothesis, we have $\Phi\left(\phi^{\prime}, C\right)=\Phi(\phi, D)$, therefore $\Phi(\phi, C)=\Phi(\phi, D)=\Phi(\psi, D)$.

(iii) Let $\left(f_{\omega}\right)_{\omega \in V \text { (Sal) }}$ be a partition of the unity subordinated to the covering $\{U(\omega) \mid \omega \in V(\mathrm{Sal})\}$ of $M(\mathscr{A})$ (see the proof of Theorem 3.3). Namely, $\left(f_{\omega}\right)_{\omega \in V(\text { Sal })}: M(\mathscr{A}) \rightarrow[0,1]$ is a collection of maps with

(1) $f_{\omega}(z)>0$ if $z \in U(\omega)$ and $f_{\omega}(z)=0$ if $z \notin U(\omega)$, for all $z \in M(\mathscr{A})$ and all $\omega \in V(\mathrm{Sal})$,

(2) $\sum_{\omega \in V(\text { Sal) }} f_{\omega}(z)=1$ for all $z \in M(\mathscr{A})$.

Let $\kappa: M(\mathscr{A}) \rightarrow \operatorname{Sal}(\mathscr{A})$ be the map defined by $\kappa(z)=\sum_{\omega \in V(\text { Sal })} f_{\omega}(z) \omega$, for all $z \in M(\mathscr{A})$. Since the covering $\{U(\omega) \mid \omega \in V(\operatorname{Sal})\}$ has $\operatorname{Sal}(\mathscr{A})$ as nerve, $\kappa$ is well defined. Moreover, by [We, p. 143], $\kappa$ is a homotopy equivalence. 
We are going to prove that $\operatorname{id}_{M(\mathscr{A})}$ and $\iota \kappa$ are homotopic. This implies that $l$ is a homotopy inverse of $\kappa$, and thus is a homotopy equivalence.

Consider the homotopy $\left(\theta_{t}\right)_{0 \leq t \leq 1}: M(\mathscr{A}) \rightarrow V_{\mathbb{C}}$ defined by

$$
\theta_{t}(z)=t z+(1-t)(\imath \kappa)(z) .
$$

Let us prove that $\theta_{t}(z) \in M(\mathscr{A})$ for all $z \in M(\mathscr{A})$ and all $t \in[0,1]$. This shows that $\left(\theta_{t}\right)_{0 \leq t \leq 1}: M(\mathscr{A}) \rightarrow M(\mathscr{A})$ is a homotopy connecting $\operatorname{id}_{M(\mathscr{A})}$ with $\imath \kappa$.

Pick $z=(x+i y) \in M(\mathscr{A})$.

Case a. Assume $x=0$.

Let $C$ be the chamber of $\mathscr{A}$ such that $y \in C$. The only vertex $\omega$ of Sal such that $z \in U(\omega)$ is $\omega=\omega(\{0\}, C)$. We have $z=(x+i y) \in(\{0\}+i C)$ and $\imath \kappa(z)=z(\{0\}, C) \in(\{0\}+i C)$, thus $\theta_{t}(z) \in(\{0\}+i C) \subseteq M(\mathscr{A})$ (since $(\{0\}+i C)$ is convex) for all $t \in[0,1]$.

Case $b$. Assume $x \neq 0$.

There is a unique simplex $\phi$ of $\mathbb{S}^{l-1}$ such that $x \in K(\phi)$.Write $\phi=x\left(F_{1}\right) \vee$ $\cdots \vee x\left(F_{r}\right)$ with $\{0\} \neq F_{1}<\cdots<F_{r}$. Set $F_{0}=\{0\}$. Since $K(\phi) \subseteq F_{r}$, there is a chamber $D_{r}$ of $\mathscr{A}_{\left|F_{r}\right|}$ with $y \in D_{r}$. On the other hand, if there is a chamber $D_{i-1}$ of $\mathscr{A}_{\left|F_{i-1}\right|}$ with $y \in D_{i-1}$, then there is a chamber $D_{i}$ of $\mathscr{A}_{\left|F_{i}\right|}$ with $y \in D_{i}$ and $D_{i-1} \subseteq D_{i}$. It follows that there exists a $j \in\{0,1, \ldots, r\}$ such that

(1) there is a chamber $D_{i}$ of $\mathscr{A}_{\left|F_{i}\right|}$ containing $y$ for all $i=j, \ldots, r$,

(2) there is no chamber of $\mathscr{A}_{\left|F_{i}\right|}$ containing $y$ for any $i=0, \ldots, j-1$,

(3) $D_{j} \subseteq D_{j+1} \subseteq \cdots \subseteq D_{r}$.

Choose a chamber $C$ of $\mathscr{A}$ such that $C_{F_{j}}=D_{j}$. We obviously have $C_{F_{i}}=$ $D_{i}$ for $i=j, \ldots, r$. The set of vertices $\omega$ of Sal such that $z \in U(\omega)$ is $\left\{\omega\left(F_{j}, C\right), \omega\left(F_{j+1}, C\right), \ldots, \omega\left(F_{r}, C\right)\right\}$. Write $\Phi=\omega\left(F_{j}, C\right) \vee \cdots \vee \omega\left(F_{r}, C\right)$. We have $z=(x+i y) \in\left(F_{r}+i C_{F_{r}}\right)$ and $l \kappa(z) \in l(\Phi) \subseteq\left(F_{r}+i C_{F_{r}}\right)$, thus $\theta_{t}(z) \in\left(F_{r}+i C_{F_{r}}\right) \subseteq M(\mathscr{A})$ (since $\left(F_{r}+i C_{F_{r}}\right)$ is convex) for all $t \in[0,1]$.

Proof of Theorem 3.7. Recall that, for every chamber $C$ of $\mathscr{A}$,

$$
q^{-1}(M(C))=\bigcup_{v \in \rho^{-1}(C)} \widehat{M}(v),
$$

this union is disjoint (Lemma 2.7), and $q$ sends $\widehat{M}(v)$ homeomorphically onto $M(C)=M(v)$ for every vertex $v \in \rho^{-1}(C)$. Furthermore, for every simplex $\phi$ of $\mathbb{B}^{l}$ and every chamber $C$ of $\mathscr{A}$, we have $l(\Phi(\phi, C)) \subseteq M(C)$ (see Lemma 4.8(i)).

Since $l: \operatorname{Sal}(\mathscr{A}) \rightarrow M(\mathscr{A})$ is injective, $l(\operatorname{Sal}(\mathscr{A}))$ can be viewed as a geometric realisation of $\operatorname{Sal}(\mathscr{A})$, so $q^{-1}(l(\operatorname{Sal}(\mathscr{A})))$ can also be seen as a geometric realization of a simplicial complex (since $q$ is a cover). Let us denote by $W$ the set of vertices of $q^{-1}(l(\operatorname{Sal}(\mathscr{A})))$. The application $\hat{\imath}: V(\widehat{\mathrm{S}} \mathrm{al}) \rightarrow W$ which sends $\hat{\omega}(F, v)$ onto $e(F, v)$, for every $F \in \mathscr{F}(\mathscr{A})$ and every $v \in V(\widehat{\text { Sal }})$, is, by the definition of $V(\widehat{\mathrm{S} a l})$, a bijection. It is easy to see that $\hat{\imath}$ can be extended to an isomorphism $\hat{\imath}$ : $\widehat{\mathrm{Sal}} \rightarrow q^{-1}(l(\operatorname{Sal}(\mathscr{A})))$ of simplicial complexes which sends $\Phi(\phi, v)$ onto the lift of $l(\Phi(\phi, C))$ into $\widehat{M}(v)$, where $C=\rho(v)$, for all simplexes $\phi$ of $\mathbb{B}^{l}$ and all vertices $v$ of $\hat{\Gamma}$. 
The following diagram commutes:

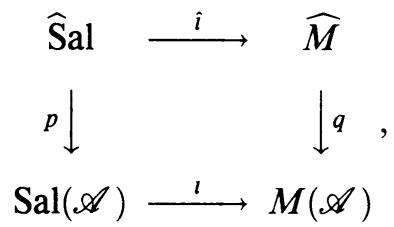

the map $q$ is a cover, $l$ and $\hat{\imath}$ are injective, and $q^{-1}(l(\operatorname{Sal}(\mathscr{A})))=\hat{\imath}(\widehat{\operatorname{Sal}})$, thus $p$ is a cover. Furthermore, $q: \widehat{M} \rightarrow M(\mathscr{A})$ is the universal cover of $M(\mathscr{A})$ and $l$ is a homotopy equivalence, thus $p: \widehat{\operatorname{Sal}} \rightarrow \operatorname{Sal}(\mathscr{A})$ is the universal cover of $\operatorname{Sal}(\mathscr{A})$ and $\hat{\imath}$ is a homotopy equivalence.

\section{TOPOLOGY OF SIMPLICIAL ARRANGEMENTS OF HYPERPLANES}

Recall that an essential arrangement $\mathscr{A}$ of hyperplanes is called simplicial if every chamber of $\mathscr{A}$ is an open simplicial cone. Our goal in this section is to prove that if $\mathscr{A}$ is a simplicial arrangement of hyperplanes, then $M(\mathscr{A})$ is a $K(\pi, 1)$ space.

This section is divided in two subsections. In the first one we define a property on real arrangements of hyperplanes: the property $D$, and we prove that if $\mathscr{A}$ is a simplicial arrangement of hyperplanes, then $\mathscr{A}$ has the property $D$ (Theorem 4.1). We do not know if, for an essential arrangement $\mathscr{A}$ of hyperplanes, to have the property $\mathrm{D}$ is equivalent to being simplicial. We will give a simple example of a supersolvable arrangement which does not have the property $D$. It is well known that, if $\mathscr{A}$ is a supersolvable arrangement of hyperplanes, then $M(\mathscr{A})$ is a $K(\pi, 1)$ space (see [Te2]).

In the second subsection we prove that if $\mathscr{A}$ has the property $D$, then $\widehat{\text { Sal }}$ is contractible (Theorem 4.6). Since Sal has the same homotopy type as the universal cover $\widehat{M}$ of $M(\mathscr{A})$ (Theorem 3.7 ), the space $\widehat{M}$ is contractible if $\mathscr{A}$ is simplicial; thus, in this case, $M(\mathscr{A})$ is a $K(\pi, 1)$ space.

4A. Property D. Throughout this subsection, $\mathscr{A}$ is an arrangement of hyperplanes in a real vector space $V$, and $(\Gamma(\mathscr{A}), \sim)$ is the oriented system associated with $\mathscr{A}$.

Let $A$ and $B$ be two chambers of $\mathscr{A}$. We say that a chamber $C$ of $\mathscr{A}$ is between $A$ and $B$ if there exists a positive minimal path $f=a_{1} \cdots a_{n}$ of $\Gamma(\mathscr{A})$ beginning at $A$, ending in $B$ and such that $C=\operatorname{end}\left(a_{1} \cdots a_{i}\right)$ for some $i=0,1, \ldots, n$. In other words, $C$ is between $A$ and $B$ if there exists a minimal gallery $\left(A=C_{0}, C_{1}, \ldots, C_{n}=B\right)$ of $\mathscr{A}$ such that $C=C_{i}$ for some $i=0,1, \ldots, n$. We denote by $\operatorname{Bet}(A, B)$ the set of chambers of $\mathscr{A}$ between $A$ and $B$.

From now on, for every pair $(A, B)$ of chambers of $\mathscr{A}$, we fix a positive minimal path $m(A, B)$ of $\Gamma(\mathscr{A})$ beginning at $A$ and ending in $B$. Note that, by the definition of the identification $\sim$ of $\Gamma(\mathscr{A})$, the equivalence class of $m(A, B)$ with respect to $\sim$ does not depend on the choice of $m(A, B)$. We obviously have $C \in \operatorname{Bet}(A, B)$ if and only if $m(A, C) m(C, B) \sim m(A, B)$.

Let $f$ and $g$ be two positive paths of $\Gamma(\mathscr{A})$ with end $(f)=\operatorname{end}(g)$. We say that $f$ ends with $g$ if there exists a positive path $h$ of $\Gamma(\mathscr{A})$ such that $f \sim h g$. 


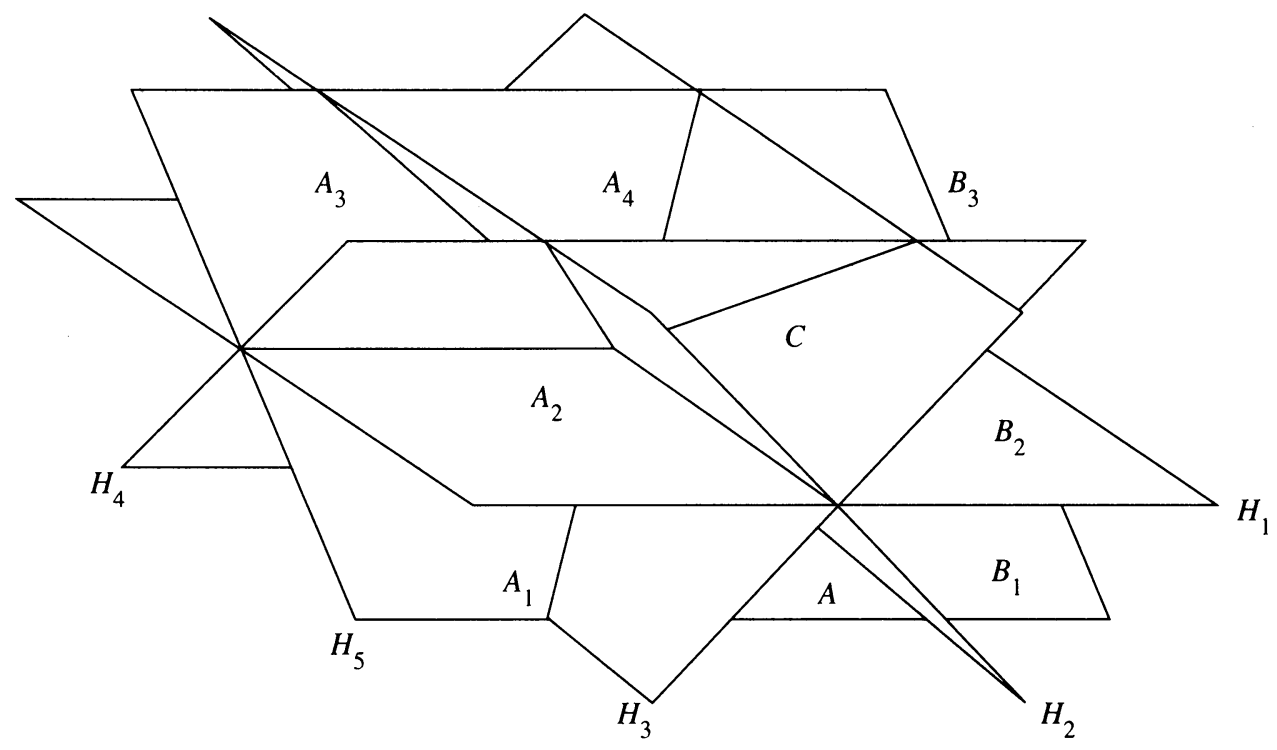

FIGURE 4

Let $f$ be a positive path of $\Gamma(\mathscr{A})$. Write $B=\operatorname{end}(f)$. We say that $f$ has the property $D$ if there exists a chamber $A$ of $\mathscr{A}$ such that $f$ ends with $m(C, B)$ if and only if $C \in \operatorname{Bet}(A, B)$, for every chamber $C$ of $\mathscr{A}$.

We say that $\mathscr{A}$ has the property $D$ if every positive path of $\Gamma(\mathscr{A})$ has the property D.

Theorem 4.1. If $\mathscr{A}$ is a simplicial arrangement of hyperplanes, then $\mathscr{A}$ has the property $D$.

Example. Consider the arrangement $\mathscr{A}=\left\{H_{1}, H_{2}, H_{3}, H_{4}, H_{5}\right\}$ in $\mathbb{R}^{3}$ shown in Figure 4. Let us show that $\mathscr{A}$ does not have the property D.

Let $f$ be the positive path of $\Gamma(\mathscr{A})$ corresponding with the gallery $\left(A, A_{1}\right.$, $\left.A_{2}, A_{3}, A_{4}, C\right)$ of $\mathscr{A}$. One can verify that $f \sim g$ and $f \sim h$, where $g$ is the positive path of $\Gamma(\mathscr{A})$ corresponding with the gallery $\left(A, A_{1}, A_{2}, A_{3}, A_{2}, C\right)$ and $h$ is the positive path of $\Gamma(\mathscr{A})$ corresponding with the gallery $\left(A, B_{1}, B_{2}\right.$, $\left.B_{3}, B_{2}, C\right)$ of $\mathscr{A}$. Thus $f$ ends with $m\left(A_{4}, C\right)$, with $m\left(A_{2}, C\right)$ and with $m\left(B_{2}, C\right)$.

Suppose that $f$ has the property $\mathrm{D}$. Then there exists a chamber $D$ of $\mathscr{A}$ such that $f$ ends with $m(B, C)$ if and only if $B \in \operatorname{Bet}(D, C)$. We have $A_{4}, A_{2}, B_{2} \in \operatorname{Bet}(D, C)$, thus the hyperplanes $H_{2}, H_{3}, H_{4}$ separate $D$ and $C$ (see Lemma 4.3), therefore $D=-C$. It is easy to see that $f$ cannot end with $M(-C, C)$ (it does not "cross" $H_{5}$ ), so we have a contradiction.

The following Lemmas 4.2-4.5 are preliminary results to the proof of Theorem 4.1. Lemmas 4.2 and 4.3 are well-known results. A proof of Lemma 4.2 and Lemma 4.3(i) can be found in [Br, p. 14]. (ii) and (iii) of Lemma 4.3 are immediate corollaries of Lemma 4.3(i).

Lemma 4.2. Let $G=\left(C_{0}, C_{1}, \ldots, C_{n}\right)$ be a gallery of $\mathscr{A}$. Let $H_{i}$ be the hyperplane of $\mathscr{A}$ which separates $C_{i-1}$ and $C_{i}$ for $i=1, \ldots, n$. The gallery $G$ is minimal if and only if $H_{i} \neq H_{j}$ for $i \neq j$. 
Corollary. Let $f=a_{1} \cdots a_{n}$ be a positive path of $\Gamma(\mathscr{A})$. Write $C_{i}=\operatorname{end}\left(a_{1} \cdots a_{i}\right)$ for $i=0,1, \ldots, n$. Let $H_{i}$ be the hyperplane of $\mathscr{A}$ which separates $C_{i-1}$ and $C_{i}$ for $i=1, \ldots, n$. The path $f$ is positive minimal if and only if $H_{i} \neq H_{j}$ for $i \neq j$.

Lemma 4.3. Let $G=\left(C_{0}, C_{1}, \ldots, C_{n}\right)$ be a minimal gallery. Let $H_{i}$ be the hyperplane of $\mathscr{A}$ which separates $C_{i-1}$ and $C_{i}$ for $i=1, \ldots, n$.

(i) The hyperplanes of $\mathscr{A}$ which separate $C_{0}$ and $C_{n}$ are exactly $H_{1}, \ldots, H_{n}$.

(ii) Let $F$ be a facet of $\mathscr{A}$. If $F$ is common to $C_{0}$ and $C_{n}$, then $H_{i}$ contains $F$ for every $i=1, \ldots, n$.

(iii) Let $F$ be a facet of $\mathscr{A}$. If $\left(C_{0}\right)_{F}=\left(C_{n}\right)_{F}$, then $H_{i}$ does not contain $F$ for any $i=1, \ldots, n$.

Corollary 1. Let $f=a_{1} \cdots a_{n}$ be a positive minimal path. Write

$$
C_{i}=\operatorname{end}\left(a_{1} \cdots a_{i}\right) \text { for } i=0,1, \ldots, n \text {. }
$$

Let $H_{i}$ be the hyperplane of $\mathscr{A}$ which separates $C_{i-1}$ and $C_{i}$ for $i=1, \ldots, n$.

(i) The hyperplanes of $\mathscr{A}$ which separate $C_{0}$ and $C_{n}$ are exactly $H_{1}, \ldots, H_{n}$.

(ii) Let $F$ be a facet of $\mathscr{A}$. If $F$ is common to $C_{0}$ and $C_{n}$, then $H_{i}$ contains $F$ for every $i=1, \ldots, n$.

(iii) Let $F$ be a facet of $\mathscr{A}$. If $\left(C_{0}\right)_{F}=\left(C_{n}\right)_{F}$, then $H_{i}$ does not contain $F$ for any $i=1, \ldots, n$.

Corollary 2. Let $A$ and $B$ be two chambers of $\mathscr{A}$. Then $m(A, B) m(B,-A)$ is a positive minimal path of $\Gamma(\mathscr{A})$.

Let $\mathscr{R}$ be the smallest equivalence relation on the set of positive paths of $\Gamma(\mathscr{A})$ such that:

(1) if $f \mathscr{R} g$, then begin $(f)=\operatorname{begin}(g)$ and end $(f)=\operatorname{end}(g)$.

(2) if $f \mathscr{R} g$, then $\left(h_{1} f h_{2}\right) \mathscr{R}\left(h_{1} g h_{2}\right)$ for any two positive paths $h_{1}$ and $h_{2}$ such that end $\left(h_{1}\right)=\operatorname{begin}(f)=\operatorname{begin}(g)$ and $\operatorname{begin}\left(h_{2}\right)=\operatorname{end}(f)=$ end $(g)$.

(3) if $f$ and $g$ are two positive minimal paths of $\Gamma(\mathscr{A})$ with the same begin and the same end, then $f \mathscr{R} g$.

Note that, if $f \mathscr{R} g$, then $f \sim g$ and length $(f)=$ length $(g)$.

Let $f$ and $g$ be two positive paths of $\Gamma(\mathscr{A})$ such that $\operatorname{end}(f)=\operatorname{end}(g)$. We say that $f \mathscr{R}$-ends with $g$ if there exists a positive path $h$ of $\Gamma(\mathscr{A})$ such that $f \mathscr{R}(h g)$.

For any chamber $A$ of $\mathscr{A}$, we write $h_{A}=m(A,-A) m(-A, A)$ and $\left(h_{A}\right)^{r}=$ $h_{A} h_{A} \cdots h_{A}$ ( $r$ times).

Lemma 4.4. Let $f$ and $g$ be two positive paths of $\Gamma(\mathscr{A})$ such that $f \sim g$. Write $A=\operatorname{begin}(f)=\operatorname{begin}(g)$. Then there exists an integer $r \geq 0$ such that $\left(h_{A}\right)^{r} f \mathscr{R}\left(h_{A}\right)^{r} g$.

Proof. We denote by $(-a)=(-A,-B)$ the opposite arrow of an arrow $a=$ $(A, B)$ of $\Gamma(\mathscr{A})$.

For every arrow $a=(A, B)$ of $\Gamma(\mathscr{A})$ set $p(a)=a$ and

$$
p\left(a^{-1}\right)=m(B,-B) m(-B, A),
$$


and for every path $f=a_{1}^{\varepsilon_{1}} \cdots a_{n}^{\varepsilon_{n}}$ set $p(f)=p\left(a_{1}^{\varepsilon_{1}}\right) \cdots p\left(a_{n}^{\varepsilon_{n}}\right)$. It is clear that $p(f)$ is a positive path of $\Gamma(\mathscr{A})$, that $p(f g)=p(f) p(g)$, and that $p(f)=f$ for every positive path $f$.

Assertion. Let $f$ and $g$ be two paths of $\Gamma(\mathscr{A})$ such that $f \sim g$. Write $A=\operatorname{begin}(f)=\operatorname{begin}(g)$. Then there exist two integers $r, s \geq 0$ such that $\left(h_{A}\right)^{r} p(f) \mathscr{R}\left(h_{A}\right)^{s} p(g)$.

This assertion proves Lemma 4.4; indeed, if $f$ and $g$ are two positive paths of $\Gamma(\mathscr{A})$ with $f \sim g$, then there exist two integers $r, s \geq 0$ such that $\left(\left(h_{A}\right)^{r} f\right) \mathscr{R}\left(\left(h_{A}\right)^{s} g\right)$. Since length $\left(\left(h_{A}\right)^{r} f\right)=$ length $\left(\left(h_{A}\right)^{s} g\right)$ and length $(f)=$ length $(g)$ (because weight $(f)=$ weight $(g))$, we have $r=s$.

Proof of the Assertion. Let $a=(A, B)$ be an arrow of $\Gamma(\mathscr{A})$. We have, by Corollary 2 of Lemma 4.3,

$$
\begin{aligned}
m(A,-A) m(-A, A) a \mathscr{R} m(A,-A)(-a) m(-B, A) a \\
\mathscr{R} m(A,-A)(-a) m(-B, B) \\
\mathscr{R} a m(B,-A)(-a) m(-B, B) \\
\mathscr{R} a m(B,-B) m(-B, B) .
\end{aligned}
$$

Thus, if $f$ is a positive path of $\Gamma(\mathscr{A})$ beginning at $A$ and ending in $B$, then $\left(\left(h_{A}\right)^{t} f\right) \mathscr{R}\left(f\left(h_{B}\right)^{t}\right)$ for every integer $t \geq 0$.

Let $f_{1}$ and $f_{2}$ be two paths of $\Gamma(\mathscr{A})$ beginning at the same chamber $A$ and ending in the same chamber $B$. Let $g$ and $h$ be two paths of $\Gamma(\mathscr{A}), g$ ending in $A$ and $h$ beginning at $B$. Write $C=\operatorname{begin}(g)$. Assume there exist two integers $r, s \geq 0$ such that $\left(\left(h_{A}\right)^{r} p\left(f_{1}\right)\right) \mathscr{R}\left(\left(h_{A}\right)^{s} p\left(f_{2}\right)\right)$. Then

$$
\begin{aligned}
\left(h_{C}\right)^{r} p\left(g f_{1} h\right) & =\left(h_{C}\right)^{r} p(g) p\left(f_{1}\right) p(h) \\
& \mathscr{R} p(g)\left(h_{A}\right)^{r} p\left(f_{1}\right) p(h) \\
& \mathscr{R} p(g)\left(h_{A}\right)^{s} p\left(f_{2}\right) p(h) \\
& \mathscr{R}\left(h_{C}\right)^{s} p\left(g f_{2} h\right) .
\end{aligned}
$$

It follows that, in order to prove the Assertion, it suffices to consider the following cases:

(a) $f$ and $g$ are positive minimal paths with the same begin and the same end.

(b) $f=f^{\prime-1}$ and $g=g^{\prime-1}$, where $f^{\prime}$ and $g^{\prime}$ are positive minimal paths with the same begin and the same end.

(c) $f=a a^{-1}$ and $g=A$, where $a=(A, B)$ is an arrow of $\Gamma(\mathscr{A})$.

(d) $f=a^{-1} a$ and $g=B$, where $a=(A, B)$ is an arrow of $\Gamma(\mathscr{A})$.

(a) Is trivial.

(b) Let $f=a_{1} \cdots a_{n}$ be a positive minimal path of $\Gamma(\mathscr{A})$. Write $A=$ begin $(f)$ and $B=\operatorname{end}(f)$. Let us prove, by induction on the length $n$ of $f$, that

$$
p\left(f^{-1}\right) \mathscr{R}\left(h_{B}\right)^{n-1} m(B,-B) m(-B, A)
$$

This clearly implies the Assertion in the case b. 
Write $A_{i}=\operatorname{end}\left(a_{1} \cdots a_{i}\right)$ for $i=1,2, \ldots, n$.

$$
\begin{aligned}
& m(-A,-B) m(-B, A) \text { is positive minimal } \\
\Rightarrow & \left(-a_{1}\right)\left(-a_{2}\right) \cdots\left(-a_{n}\right) m(-B, A) \text { is positive minimal } \\
\Rightarrow & \left(-a_{2}\right) \cdots\left(-a_{n}\right) m(-B, A) \text { is positive minimal } \\
\Rightarrow & m\left(-A_{1},-B\right) m(-B, A) \text { is positive minimal } \\
\Rightarrow & m\left(-A_{1},-B\right) m(-B, A) \mathscr{R} m\left(-A_{1}, A\right) .
\end{aligned}
$$

It follows that

$$
\begin{aligned}
& p\left(f^{-1}\right)= p\left(a_{n}^{-1} \cdots a_{2}^{-1}\right) p\left(a_{1}^{-1}\right) \\
& \mathscr{R}\left(h_{B}\right)^{n-2} m(B,-B) m\left(-B, A_{1}\right) m\left(A_{1},-A_{1}\right) m\left(-A_{1}, A\right) \\
& \mathscr{R}\left(h_{B}\right)^{n-2} m(B,-B) m\left(-B, A_{1}\right) m\left(A_{1}, B\right) m\left(B,-A_{1}\right) \\
& \quad m\left(-A_{1},-B\right) m(-B, A) \\
& \mathscr{R}\left(h_{B}\right)^{n-2} m(B,-B) m(-B, B) m(B,-B) m(-B, A) \\
& \mathscr{R}\left(h_{B}\right)^{n-1} m(B,-B) m(-B, A) .
\end{aligned}
$$

(c)

$$
\begin{aligned}
p(f) & =\operatorname{am}(B,-B) m(-B, A) \\
& \mathscr{R} \operatorname{am}(B,-A)(-a) m(-B, A) \\
& \mathscr{R} m(A,-A) m(-A, A)=h_{A}=h_{A} p(g) .
\end{aligned}
$$

(d)

$$
\begin{array}{rl}
p(f) & =m(B,-B) m(-B, A) a \\
\mathscr{R} & m(B,-B) m(-B, B)=h_{B} p(g) .
\end{array}
$$

Lemma 4.5. Assume $\mathscr{A}$ to be simplicial.

(i) Let $f_{1}, f_{2}$ and $g$ be three positive paths of $\Gamma(\mathscr{A})$ such that end $(g)=$ $\operatorname{begin}\left(f_{1}\right)=\operatorname{begin}\left(f_{2}\right)$. If $\left(g f_{1}\right) \mathscr{R}\left(g f_{2}\right)$, the $f_{1} \mathscr{R} f_{2}$.

(ii) Let $f_{1}, f_{2}$ and $h$ be three positive paths of $\Gamma(\mathscr{A})$ with begin $(h)=$ end $\left(f_{1}\right)=\operatorname{end}\left(f_{2}\right)$. If $\left(f_{1} h\right) \mathscr{R}\left(f_{2} h\right)$, then $f_{1} \mathscr{R} f_{2}$.

(iii) Let $f$ be a positive path of $\Gamma(\mathscr{A})$. Write $B=\operatorname{end}(f)$. There exists a chamber $A$ of $\mathscr{A}$ such that $f \mathscr{R}$-ends with $m(C, B)$ if and only if $C \in$ $\operatorname{Bet}(A, B)$.

Proof. See [De, Proposition 1.19].

Proof of Theorem 4.1. Assume $\mathscr{A}$ to be simplicial. Let $f$ and $g$ be two positive paths of $\Gamma(\mathscr{A})$. If $f \mathscr{R} g$, then obviously $f \sim g$. On the other hand, if $f \sim g$, then there exists an integer $r \geq 0$ such that $\left(\left(h_{A}\right)^{r} f\right) \mathscr{R}\left(\left(h_{A}\right)^{r} g\right)$ (Lemma 4.4), where $A=\operatorname{begin}(f)=\operatorname{begin}(g)$, therefore, by Lemma 4.5(i), $f \mathscr{R} g$. Thus $f \sim g$ if and only if $f \mathscr{R} g$. In particular, a positive path $f$ of $\Gamma(\mathscr{A})$ ends with a positive path $g$ if and only if $f \mathscr{R}$-ends with $g$. Then Theorem 4.1 easily follows from Lemma 4.5(iii).

4.B. Property $D$ and the topology of $\widehat{S}$ al. Throughout this subsection, $\mathscr{A}$ is an essential arrangement of hyperplanes, $\rho:(\widehat{\Gamma}, \sim) \rightarrow(\Gamma(\mathscr{A}), \sim)$ is the universal cover of the oriented system $(\Gamma(\mathscr{A}), \sim)$ associated with $\mathscr{A}$, and $p: \widehat{\operatorname{Sal}} \rightarrow \operatorname{Sal}(\mathscr{A})$ is the universal cover of Salvetti's complex $\operatorname{Sal}(\mathscr{A})$ as defined in $\S 3$. 
Theorem 4.6. If $\mathscr{A}$ has the property $D$, then $\widehat{\text { Sal }}$ is contractible.

Corollary. If $\mathscr{A}$ is a simplicial arrangement of hyperplanes, then $M(\mathscr{A})$ is a $K(\pi, 1)$ space.

The following Lemmas 4.7-4.12 are preliminary results to the proof of Theorem 4.6.

Lemma 4.7. Let $X$ be a simplicial complex. We denote by $V(X)$ the set of vertices of $X$. Let $W \subseteq V(X)$ be a subset. Let $Y$ be the subcomplex of $X$ generated by $W$ (i.e., $Y$ is the union of the simplexes of $X$ having their vertices in $W$ ), and let $Z$ be the subcomplex of $X$ generated by $V(X)-W$.

Then $Y$ is a strong deformation retract of $(X-Z)$.

Proof. We have to define a continuous family $\left(\theta_{t}\right)_{0 \leq t \leq 1}:(X-Z) \rightarrow(X-Z)$ of maps such that

(1) $\theta_{0}(x)=x$, for all $x \in(X-Z)$,

(2) $\theta_{1}(x) \in Y$, for all $x \in(X-Z)$,

(3) $\theta_{t}(x)=x$, for all $x \in Y$ and all $t \in[0,1]$.

Let $\Phi$ be a simplex of $X$ included in $(X-Z)$. Let $\omega_{0}, \omega_{1}, \ldots, \omega_{r}$ be the vertices of $\Phi$. Via the canonical embedding $\Phi \rightarrow \mathbb{R}^{r+1}$, every element $x \in \Phi$ can be written in a unique way

$$
x=\sum_{i=0}^{r} t_{i} \omega_{i},
$$

with $0<t_{i} \leq 1$ for $i=0,1, \ldots, r$, and $\sum_{i=0}^{r} t_{i}=1$. Since $\Phi \subseteq(X-Z)$, there is at least one vertex of $\Phi$ included in $W$. Assume $\omega_{0}, \omega_{1}, \ldots, \omega_{s}$ to be the vertices of $\Phi$ included in $W$. The restriction of $\theta_{t}$ to $\Phi$ is defined by

$$
\theta_{t}\left(\sum_{i=0}^{r} t_{i} \omega_{i}\right)=t\left(\sum_{i=0}^{r} t_{i} \omega_{i}\right)+(1-t)\left(\sum_{i=0}^{s} t_{i}\right)^{-1}\left(\sum_{i=0}^{s} t_{i} \omega_{i}\right) .
$$

It is clear that $\theta_{t}$ is well defined and satisfies (1), (2), and (3).

A wall of a chamber $A$ of $\mathscr{A}$ is the support of a face of $A$ (i.e., of a 1 codimension facet of $A$ ).

Lemma 4.8. Let $A$ be a chamber of $\mathscr{A}$, and let $H_{1}, \ldots, H_{r}$ be $r$ distinct walls of $A$. Consider the subcomplex $\Delta$ of $\mathbb{S}^{l-1}$ generated by the vertices $x(F)$ of $\mathbb{S}^{l-1}$ included in $\bigcup_{i=1}^{r}\left(H_{i}\right)_{A}^{+}$. Then $\Delta$ is a strong deformation retract of $\mathbb{B}^{l}$.

Proof. Apply Lemma 4.7 to $X=\mathbb{S}^{l-1}$ and $W$ the set of vertices $x(F)$ of $\mathbb{S}^{l-1}$ included in $\bigcup_{i=1}^{r}\left(H_{i}\right)_{A}^{+}$. We have $X-Z=\mathbb{S}^{l-1} \cap\left(\bigcup_{i=1}^{r}\left(H_{i}\right)_{A}^{+}\right)$and $Y=\Delta$. It follows that $\Delta$ is a strong deformation retract of $\mathbb{S}^{l-1} \cap\left(\bigcup_{i=1}^{r}\left(H_{i}\right)_{A}^{+}\right)$. Since $\mathbb{S}^{l-1} \cap\left(\bigcup_{i=1}^{r}\left(H_{i}\right)_{A}^{+}\right)=\mathbb{S}^{l-1}-\left(\bigcap_{i=1}^{r}\left(\bar{H}_{i}\right)_{A}^{-}\right)$is contractible (where $\left(\bar{H}_{i}\right)_{A}^{-}$is the closed half-space of $V$ bordered by $H_{i}$ and not containing $A$ ), the subcomplex $\Delta$ is contractible, thus is a strong deformation retract of $\mathbb{B}^{l}$ (see [LW, Theorem 3.1, Chapter IV], for example).

Fix a vertex $v_{0}$ of $\widehat{\Gamma}$. We denote by $V(\widehat{\Gamma})_{+}\left(v_{0}\right)=V(\widehat{\Gamma})_{+}$the set of vertices $v$ of $\widehat{\Gamma}$ such that there exists a positive path in $\widehat{\Gamma}$ beginning at $v_{0}$ and ending in $v$. We denote by $V(\widehat{\Gamma})_{n}\left(v_{0}\right)=V(\widehat{\Gamma})_{n}$ the set of vertices $v$ of $\widehat{\Gamma}$ such that there 
exists a positive path in $\widehat{\Gamma}$ of length $\leq n$ beginning at $v_{0}$ and ending in $v$. Note that, if $f$ and $g$ are both positive paths of $\widehat{\Gamma}$ beginning at $v_{0}$ and ending in $v \in V(\widehat{\Gamma})_{+}$, then $f \sim g$ (Proposition 2.2), thus length $(f)=\operatorname{length}(g)$. For $v \in V(\widehat{\Gamma})_{+}$, we denote by $d\left(v_{0}, v\right)$ the length of a positive path of $\widehat{\Gamma}$ beginning at $v_{0}$ and ending in $v$.

We denote by $\widehat{S}_{+} l_{+}\left(v_{0}\right)=\widehat{S} \mathrm{al}_{+}$the subcomplex of $\widehat{\mathrm{S} a l}$ generated by the

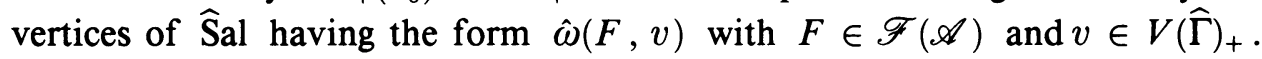
We have

$$
\widehat{\mathrm{Sal}}_{+}=\bigcup_{v \in V(\widehat{\Gamma})_{+}} \mathbb{B}^{l}(v) .
$$

We denote by $\widehat{S} \mathrm{al}_{n}\left(v_{0}\right)=\widehat{\mathrm{S}} \mathrm{a}_{n}$ the subcomplex of $\widehat{\mathrm{S}} \mathrm{al}$ generated by the vertices of Sal having the form $\hat{\omega}(F, v)$ with $F \in \mathscr{F}(\mathscr{A})$ and $v \in V(\widehat{\Gamma})_{n}$. We have

$$
\widehat{\mathrm{Sal}} \mathrm{l}_{n}=\bigcup_{v \in V(\widehat{\Gamma})_{n}} \mathbb{B}^{l}(v) .
$$

Lemma 4.9. Assume $\mathscr{A}$ to have the property $D$. Fix a vertex $v_{0}$ of $\widehat{\Gamma}$. Let $v, w \in V(\widehat{\Gamma})_{n+1}-V(\widehat{\Gamma})_{n}$ with $v \neq w$. Then

$$
\mathbb{B}^{l}(v) \cap B^{l}(w) \subseteq \widehat{\mathrm{Sal}}_{n} .
$$

Proof. Let $\hat{\omega}$ be a vertex of Sal included in $\mathbb{B}^{l}(v) \cap \mathbb{B}^{l}(\omega)$. Write $\rho(v)=A$ and $\rho(w)=B$. There exist two facets $F$ and $G$ of $\mathscr{A}$ such that $\hat{\omega}=\hat{\omega}(F, v)=$ $\hat{\omega}(G, w)$. By Lemma 3.4, $F=G \subseteq Z(v, w)$ and $A_{F}=B_{F}$.

Let $C$ be the chamber of $\mathscr{A}$ such that $F$ is a facet of $C$ and $C_{F}=-A_{F}=$ $-B_{F}$. The set $Z(v, w)$ is a union of facets of $\mathscr{A}$, it is an open subset of $V$, $F \subseteq Z(v, w)$, and $C \geq F$, thus $C \subseteq Z(v, w)$. Therefore there exists a vertex $u \in \Sigma(v) \cap \Sigma(w)$ such that $\rho(u)=C$.

Let $m(v, u)$ be a positive path of $\widehat{\Gamma}$ beginning at $v$ and ending in $u$, and let $m(w, u)$ be a positive path of $\widehat{\Gamma}$ beginning at $w$ and ending in $u$. Since $u \in \Sigma(v) \cap \Sigma(w)$, one can assume $\rho(m(v, u))=m(A, C)$ and $\rho(m(w, u))=$ $m(B, C)$.

Pick a positive path $\hat{f}$ of $\widehat{\Gamma}$ beginning at $v_{0}$ and ending in $v$, and a positive path $\hat{g}$ of $\hat{\Gamma}$ beginning at $v_{0}$ and ending in $w$. Write

(i) $f=\rho(\hat{f})$ and $g=\rho(\hat{g})$,

(ii) $\hat{f}_{0}=\hat{f} m(v, u)$ and $\hat{g}_{0}=\hat{g} m(w, u)$,

(iii) $f_{0}=\rho\left(\hat{f}_{0}\right)=f m(A, C)$ and $g_{0}=\rho\left(\hat{g}_{0}\right)=g m(B, C)$.

Note that $\hat{f}_{0}$ and $\hat{g}_{0}$ have the same begin $v_{0}$ and the same end $u$, thus $\hat{f}_{0} \sim \hat{g}_{0}$ (Proposition 2.2), therefore $f_{0} \sim g_{0}$.

Recall that $\mathscr{A}$ has the property D. There exists a chamber $C_{0}$ of $\mathscr{A}$ such that $f_{0}$ ends with $m(D, C)$ if and only if $D \in \operatorname{Bet}\left(C_{0}, C\right)$. Choose a positive path $h$ of $\Gamma(\mathscr{A})$ such that $f_{0} \sim h m\left(C_{0}, C\right)$. Let $\hat{h}$ be the lift of $h$ into $\widehat{\Gamma}$ beginning at $v_{0}$. Write $u_{0}=\operatorname{end}(\hat{h})$.

Let us prove that $u_{0} \in V(\widehat{\Gamma})_{n}$ and that $\hat{\omega}=\hat{\omega}\left(F, u_{0}\right)$. This shows that $\hat{\omega} \in \widehat{\mathrm{Sal}}_{n}$, thus ends the proof of Lemma 4.9. 
First, let us prove that $C_{0} \neq A$. If not, then

$$
\begin{aligned}
n+1 & =\text { length }(f) \quad\left(\operatorname{since} v \in V(\widehat{\Gamma})_{n+1}-V(\widehat{\Gamma})_{n}\right) \\
= & \text { length }\left(f_{0}\right)-\text { length }\left(m\left(C_{0}, C\right)\right) \quad\left(\text { since } C_{0}=A\right) \\
= & \text { length }\left(f_{0}\right)-\text { length }\left(m\left(C_{0}, B\right)\right)-\text { length }(m(B, C)) \\
& \quad\left(\text { since } B \in \operatorname{Bet}\left(C_{0}, C\right)\right) \\
= & \text { length }(g)-\operatorname{length}\left(m\left(C_{0}, B\right)\right) \quad\left(\text { since } f_{0} \sim g_{0}\right) \\
= & n+1-\text { length }\left(m\left(C_{0}, B\right)\right) \quad\left(\text { since } w \in V(\widehat{\Gamma})_{n+1}-V(\widehat{\Gamma})_{n}\right) .
\end{aligned}
$$

It follows that $A=C_{0}=B$, thus $m(A, C)=m(B, C)$, therefore $m(v, u)=$ $m(w, u)$. This contradicts the fact that $v \neq w$.

Now,

$$
\begin{aligned}
d\left(v_{0}, u_{0}\right) & =\text { length }(\hat{h}) \\
& =\text { length }(h) \\
& =\text { length }\left(f_{0}\right)-\text { length }\left(m\left(C_{0}, C\right)\right) \quad\left(\operatorname{since} f_{0} \sim h m\left(C_{0}, C\right)\right) \\
& =\text { length }\left(f_{0}\right)-\text { length }\left(m\left(C_{0}, A\right)\right)-\operatorname{length}(m(A, C)) \\
& =\quad\left(\operatorname{since} A \in \operatorname{Bet}\left(C_{0}, C\right)\right) \\
& =n+1-\text { length }(f)-\text { length }\left(m\left(C_{0}, A\right)\right) \quad\left(\text { since } v \in V(\widehat{\Gamma})_{n+1}-V\left(C_{0}, A\right)\right) \\
& \leq n \quad\left(\operatorname{since} C_{0} \neq A\right) .
\end{aligned}
$$

This shows that $u_{0} \in V(\widehat{\Gamma})_{n}$.

If $H \supseteq F$, then $H$ separates $A$ and $C$ (since $C_{F}=-A_{F}$ ), thus $H$ does not separate $C_{0}$ and $A$ (since $A \in \operatorname{Bet}\left(C_{0}, C\right)$ ), therefore $\left(C_{0}\right)_{F}=A_{F}$. The vertex $u$ is included in $\Sigma(v) \cap \Sigma\left(u_{0}\right)$ (lift $m\left(C_{0}, A\right) m(A, C)$ into $\left.\widehat{\Gamma}\right)$, thus $\rho(u)=C \subseteq Z\left(v, u_{0}\right)$. Since $F \subseteq \bar{C}$, we have $F \subseteq \bar{Z}\left(v, u_{0}\right)$. If $H$ separates $A=\rho(v)$ and $C_{0}=\rho\left(u_{0}\right)$, then $H$ does not contain $F$ (since $\left.\left(C_{0}\right)_{F}=A_{F}\right)$. It follows that $F$ is not included in the border of $Z\left(v, u_{0}\right)$ (see Lemma 2.5), thus $F \subseteq Z\left(v, u_{0}\right)$. By Lemma 3.4, we have $\hat{\omega}=\hat{\omega}(F, v)=\hat{\omega}\left(F, u_{0}\right)$.

Lemma 4.10. Assume $\mathscr{A}$ to have the property $D$. Fix a vertex $v_{0}$ of $\widehat{\Gamma}$. Let $v \in V(\widehat{\Gamma})_{n+1}-V(\widehat{\Gamma})_{n}$. Write $A=\rho(v)$. Then there exists a set $\left\{H_{1}, \ldots, H_{r}\right\}$ of walls of $A$ such that $\mathbb{B}^{l}(v) \cap \widehat{\mathrm{Sal}}_{n}$ is the subcomplex of $\mathbb{B}^{l}(v)$ generated by the vertices of $\mathbb{B}^{l}(v)$ having the form $\hat{\omega}(F, v)$ with $F \subseteq \bigcup_{i=1}^{r}\left(H_{i}\right)_{A}^{+}$.

Proof. Choose a positive path $\hat{f}$ of $\widehat{\Gamma}$ beginning at $v_{0}$ and ending in $v$. Write $f=\rho(\hat{f})$. Let $a_{1}, \ldots, a_{r}$ be all the arrows of $\Gamma(\mathscr{A})$ such that $f$ ends with $a_{i}$ (for $i=1, \ldots, r)$. Write $A_{i}=\operatorname{begin}\left(a_{i}\right)$ and $H_{i}$ the hyperplane of $\mathscr{A}$ which separates $A_{i}$ and $A$, for $i=1, \ldots, r$. Let us show that $\left\{H_{1}, \ldots, H_{r}\right\}$ is the required set of walls of $A$.

Let $F$ be a facet of $\mathscr{A}$. Assume there exists an $i \in\{1, \ldots, r\}$ such that $F \subseteq\left(H_{i}\right)_{A}^{+}$. Since $f$ ends with $a_{i}$, there exists a positive path $h$ of $\Gamma(\mathscr{A})$ such that $f \sim h a_{i}$. Let $\hat{h}$ be the lift of $h$ into $\widehat{\Gamma}$ beginning at $v_{0}$. Write $w_{i}=\operatorname{end}(\hat{h})$. Let us prove that $w_{i} \in V(\widehat{\Gamma})_{n}$ and that $\hat{\omega}(F, v)=\hat{\omega}\left(F, w_{i}\right)$. This shows that $\hat{\omega}(F, v) \in \mathbb{B}^{l}(v) \cap \widehat{\mathrm{S}} \mathrm{a}_{n}$.

thus $w_{i} \in V(\widehat{\Gamma})_{n}$.

$$
d\left(v_{0}, w_{i}\right)=\operatorname{length}(\hat{h})=\text { length }(h)=n,
$$


Since $F \subseteq\left(H_{i}\right)_{A}^{+}$, the facet $F$ is not included in $H_{i}$, thus $\left(A_{i}\right)_{F}=A_{F}$. Pick a chamber $C$ of $\mathscr{A}$ having $F$ as facet. Since $F \subseteq\left(H_{i}\right)_{A}^{+}$and $F \subseteq \bar{C}$, we have $C \subseteq\left(H_{i}\right)_{A}^{+}$, thus $H_{i}$ does not separate $A$ and $C$, therefore $A \in$ $\operatorname{Bet}\left(A_{i}, C\right)$ (by Lemmas 4.2 and 4.3(i)). This implies that there exists a vertex $u \in \Sigma(v) \cap \Sigma\left(w_{i}\right)$ such that $\rho(u)=C$ (lift $m\left(A_{i}, A\right) m(A, C)$ into $\left.\widehat{\Gamma}\right)$. It follows that $C \subseteq Z\left(v, w_{i}\right)$, therefore $F \subseteq \bar{Z}\left(v, w_{i}\right)$ (since $F \subseteq \bar{C}$ ). The equality $\left(A_{i}\right)_{F}=A_{F}$ shows that no hyperplane of $\mathscr{A}$ containing $F$ separates $A_{i}=\rho\left(w_{i}\right)$ and $A=\rho(v)$, thus, by Lemma 2.5 , we have $F \subseteq Z\left(v, w_{i}\right)$. It follows, by Lemma 3.4, that $\hat{\omega}(F, v)=\hat{\omega}\left(F, w_{i}\right)$.

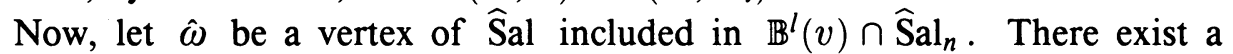
vertex $w \in V(\widehat{\Gamma})_{n}$ and two facets $F$ and $G$ of $\mathscr{A}$ such that $\hat{\omega}=\hat{\omega}(F, v)=$ $\hat{\omega}(G, w)$. Write $B=\rho(w)$. By Lemma 3.4, $F=G \subseteq Z(v, w)$ and $A_{F}=B_{F}$.

Let $C$ be the chamber of $\mathscr{A}$ having $F$ as facet and such that $C_{F}=-A_{F}=$ $-B_{F}$. The set $Z(v, w)$ is a union of facets of $\mathscr{A}$, it is an open subset of $V, F \subseteq Z(v, w)$, and $C \geq F$, thus $C \subseteq Z(v, w)$. Therefore there exists a vertex $u \in \Sigma(v) \cap \Sigma(w)$ such that $\rho(u)=C$. Let $m(v, u)$ be a positive path of $\widehat{\Gamma}$ beginning at $v$ and ending in $u$, and let $m(w, u)$ be a positive path of $\widehat{\Gamma}$ beginning at $w$ and ending in $u$. Since $u \in \Sigma(v) \cap \Sigma(w)$, one can assume $\rho(m(v, u))=m(A, C)$ and $\rho(m(w, u))=m(B, C)$.

Pick a positive path $\hat{f}$ of $\hat{\Gamma}$ beginning at $v_{0}$ and ending in $v$, and a positive path $\hat{g}$ of $\hat{\Gamma}$ beginning at $v_{0}$ and ending in $w$. Write

(i) $f=\rho(\hat{f})$ and $g=\rho(\hat{g})$,

(ii) $\hat{f}_{0}=\hat{f} m(v, u)$ and $\hat{g}_{0}=\hat{g} m(w, u)$,

(iii) $f_{0}=\rho\left(\hat{f}_{0}\right)=f m(A, C)$ and $g_{0}=\rho\left(\hat{g}_{0}\right)=g m(B, C)$.

Note that $\hat{f}_{0}$ and $\hat{g}_{0}$ have the same begin $v_{0}$ and the same end $u$, thus $\hat{f}_{0} \sim \hat{g}_{0}$ (Proposition 2.2), therefore $f_{0} \sim g_{0}$.

Recall that $\mathscr{A}$ has the property $\mathrm{D}$. There exists a chamber $C_{0}$ of $\mathscr{A}$ such that $f_{0}$ ends with $m(D, C)$ if and only if $D \in \operatorname{Bet}\left(C_{0}, C\right)$. Choose a positive path $h$ of $\Gamma(\mathscr{A})$ such that $f_{0} \sim h m\left(C_{0}, C\right)$. As in the proof of Lemma 4.9, $f_{0} \sim g_{0}$ and $v \neq w$ imply $C_{0} \neq A$. Since

$$
f_{0}=f m(A, C) \sim h m\left(C_{0}, A\right) m(A, C)
$$

indeed, $\left.A \in \operatorname{Bet}\left(C_{0}, C\right)\right)$, the path $f$ ends with $m\left(C_{0}, A\right)$.

Write $m\left(C_{0}, A\right)=b_{1} \cdots b_{n}$. There is an $i \in\{1, \ldots, r\}$ such that $b_{n}=a_{i}$. Since $A \in \operatorname{Bet}\left(C_{0}, C\right)$ and $H_{i}$ separates $C_{0}$ and $A$, the hyperplane $H_{i}$ does not separate $A$ and $C$ (by Lemmas 4.2 and 4.3(i)), thus $C \subseteq\left(H_{i}\right)_{A}^{+}$. This implies $F \subseteq\left(\bar{H}_{i}\right)_{A}^{+}$(since $F \subseteq \bar{C}$ ), where $\left(\bar{H}_{i}\right)_{A}^{+}$is the closed half-space of $V$ bordered by $H_{i}$ and containing $A$. If $H \in \mathscr{A}$ contains $F$, then $H$ separates $A$ and $C$ (since $C_{F}=-A_{F}$ ), thus $H$ does not separate $C_{0}$ and $A$ (since $\left.A \in \operatorname{Bet}\left(C_{0}, C\right)\right)$, therefore $H \neq H_{i}$. It follows that $F \subseteq\left(H_{i}\right)_{A}^{+}$.

Lemma 4.11. Assume $\mathscr{A}$ to have the property $D$. Fix a vertex $v_{0}$ of $\widehat{\Gamma}$. Then $\widehat{\mathrm{Sal}}{ }_{+}\left(v_{0}\right)$ is contractible.

Proof. Let us show that $\widehat{S}_{a} l_{n}$ is a strong deformation retract of $\widehat{S}_{a} a_{n+1}$ for all $n \geq 0$. Since $\widehat{\mathrm{Sal}}_{+}=\underline{\lim } \widehat{\mathrm{Sal}}_{n}$ and $\widehat{\mathrm{Sal}}_{0}=\mathbb{B}^{l}\left(v_{0}\right)$ is contractible, this proves Lemma 4.11 . 
We have to define a continuous family $\left(\theta_{t}\right)_{0 \leq t \leq 1}: \widehat{\operatorname{Sal}}_{n+1} \rightarrow \widehat{\mathrm{Sal}}_{n+1}$ of maps such that

(1) $\theta_{0}(x)=x$, for al $x \in \widehat{S}_{\mathrm{Sa}} \mathrm{l}_{n+1}$,

(2) $\theta_{1}(x) \in \widehat{S}_{\mathrm{al}_{n}}$, for all $x \in \widehat{\mathrm{Sal}}_{n+1}$,

(3) $\theta_{t}(x)=x$, for all $x \in \widehat{\mathrm{Sa}}_{n}$ and all $t \in[0,1]$.

Let $v \in V(\widehat{\Gamma})_{n+1}-V(\widehat{\Gamma})_{n}$. Write $A=\rho(v)$. By Lemma 4.10 , there exists a set $\left\{H_{1}, \ldots, H_{r}\right\}$ of walls of $A$ such that $\hat{\pi}_{v}\left(\mathbb{B}^{l}(v) \cap \widehat{S}_{a} a_{n}\right)$ is the subcomplex of $\mathbb{B}^{l}$ generated by the vertices $x(F)$ of $\mathbb{B}^{l}$ included in $\bigcup_{i=1}^{r}\left(H_{i}\right)_{A}^{+}$. Note that $x(\{0\})=0 \notin \bigcup_{i=1}^{r}\left(H_{i}\right)_{A}^{+}$. By Lemma 4.8, $\hat{\pi}_{v}\left(\mathbb{B}^{l}(v) \cap \widehat{\mathrm{Sal}}{ }_{n}\right)$ is a strong deformation retract of $\mathbb{B}^{l}$. It follows that $\mathbb{B}^{l}(v) \cap \widehat{\mathrm{Sa}}{ }_{n}$ is a strong deformation retract of $\mathbb{B}^{l}(v)$ (since $\hat{\pi}_{v}: \mathbb{B}^{l}(v) \rightarrow \mathbb{B}^{l}$ is an isomorphism). Choose a homotopy $\left(\theta_{t}^{v}\right)_{0 \leq t \leq 1}: \mathbb{B}^{l}(v) \rightarrow \mathbb{B}^{l}(v)$ with

(a) $\theta_{0}^{v}(x)=x$, for all $x \in \mathbb{B}^{l}(v)$,

(b) $\theta_{1}^{v}(x) \in \mathbb{B}^{l}(v) \cap \widehat{\mathrm{S}} \mathrm{a}_{n}$, for all $x \in \mathbb{B}^{l}(v)$,

(c) $\theta_{t}^{v}(x)=x$, for all $x \in \mathbb{B}^{l}(v) \cap \widehat{\mathrm{Sa}} \mathrm{l}_{n}$ and all $t \in[0,1]$.

Set $\left.\theta_{t}\right|_{\mathbb{B}^{\prime}(v)}=\theta_{t}^{v}$ if $v \in V(\widehat{\Gamma})_{n+1}-V(\widehat{\Gamma})_{n}$, and $\left.\theta_{t}\right|_{\mathbb{B}^{\prime}(v)}=\mathrm{id}_{\mathbb{B}^{\prime}(v)}$ if $v \in V(\widehat{\Gamma})_{n}$.

By Lemma 4.9 , the homotopy $\theta_{t}$ is well defined. It obviously satisfies (1), (2) and (3).

Recall that, for a chamber $A$ of $\mathscr{A}, h_{A}=m(A,-A) m(-A, A)$. Write $\left(h_{A}\right)^{-r}=\left(h_{A}\right)^{-1}\left(h_{A}\right)^{-1} \cdots\left(h_{A}\right)^{-1}(r$ times $)$ for all $r \geq 0$.

Lemma 4.12. Let $f$ be a path of $\Gamma(\mathscr{A})$. Write $A=\operatorname{begin}(f)$. Then there exist a positive path $g$ of $\Gamma(\mathscr{A})$ and an integer $r \geq 0$ such that $f \sim\left(h_{A}\right)^{-r} g$.

Proof. Write $f=a_{1}^{\varepsilon_{1}} \cdots a_{n}^{\varepsilon_{n}}$. Set $r=\left|\left\{i \in\{1, \ldots, r\} \mid \varepsilon_{i}=-1\right\}\right|$. We are going to prove, by induction on $r$, that there exists a positive path $g$ of $\Gamma(\mathscr{A})$ such that $f \sim\left(h_{A}\right)^{-r} g$.

Assume $r>0$. For an arrow $a=(C, D)$ of $\Gamma(\mathscr{A})$, we write $(-a)=$ $(-C,-D)$. Let $A_{i}=\operatorname{end}\left(a_{1}^{\varepsilon_{1}} \cdots a_{i}^{\varepsilon_{i}}\right)$ for $i=1,2, \ldots, n$. There exists a $j \in\{1, \ldots, n\}$ such that $\varepsilon_{1}=\varepsilon_{2}=\cdots=\varepsilon_{j-1}=1$ and $\varepsilon_{j}=-1$. We have

$$
\begin{aligned}
h_{A} f & \sim m(A,-A) m(-A, A) a_{1} \cdots a_{j-1} a_{j}^{-1} a_{j+1}^{\varepsilon_{j+1}} \cdots a_{n}^{\varepsilon_{n}} \\
\sim & m(A,-A)\left(-a_{1}\right) m\left(-A_{1}, A\right) a_{1} a_{2} \cdots a_{j-1} a_{j}^{-1} a_{j+1}^{\varepsilon_{j+1}} \cdots a_{n}^{\varepsilon_{n}} \\
\sim & m(A,-A)\left(-a_{1}\right) m\left(-A_{1}, A_{1}\right) a_{2} \cdots a_{j-1} a_{j}^{-1} a_{j+1}^{\varepsilon_{j+1}} \cdots a_{n}^{\varepsilon_{n}} \\
& \cdots \\
& \sim m(A,-A)\left(-a_{1}\right)\left(-a_{2}\right) \cdots\left(-a_{j-1}\right) m\left(-A_{j-1}, A_{j-1}\right) a_{j}^{-1} a_{j+1}^{\varepsilon_{j+1}} \cdots a_{n}^{\varepsilon_{n}} \\
& \sim m(A,-A)\left(-a_{1}\right) \cdots\left(-a_{j-1}\right) m\left(-A_{j-1}, A_{j}\right) a_{j} a_{j}^{-1} a_{j+1}^{\varepsilon_{j+1}} \cdots a_{n}^{\varepsilon_{n}} \\
& \sim m(A,-A)\left(-a_{1}\right) \cdots\left(-a_{j-1}\right) m\left(-A_{j-1}, A_{j}\right) a_{j+1}^{\varepsilon_{j+1}} \cdots a_{n}^{\varepsilon_{n}} \\
& \sim\left(h_{A}\right)^{1-r} g \quad \text { (inductive hypothesis), }
\end{aligned}
$$

where $g$ is a positive path. Thus $f \sim\left(h_{A}\right)^{-r} g$.

Proof of Theorem 4.6. Fix a vertex $v_{0} \in V(\widehat{\Gamma})$. Write $A=\rho\left(v_{0}\right)$. Let us define, by induction on $r>0$, a vertex $v_{r} \in V(\widehat{\Gamma})$ with $\rho\left(v_{r}\right)=A$. Assume $v_{r-1}$ to be defined. Let $\hat{h}_{r}$ be the lift of $h_{A}$ into $\widehat{\Gamma}$ ending in $v_{r-1}$. We set $v_{r}=\operatorname{begin}\left(\hat{h}_{r}\right)$. 
We clearly have $V(\widehat{\Gamma})_{+}\left(v_{r-1}\right) \subseteq V(\widehat{\Gamma})_{+}\left(v_{r}\right)$, thus $\widehat{\operatorname{Sal}}_{+}\left(v_{r-1}\right) \subseteq \widehat{\mathrm{Sal}}_{+}\left(v_{r}\right)$, for $r>0$.

Let us prove that, for every vertex $w \in V(\widehat{\Gamma})$, there exists an integer $r \geq 0$ such that $w \in V(\widehat{\Gamma})_{+}\left(v_{r}\right)$. This shows that

$$
\widehat{\mathrm{Sal}}=\bigcup_{w \in V(\widehat{\Gamma})} \mathbb{B}^{l}(w)=\underline{\lim } \widehat{\mathrm{Sal}}_{+}\left(v_{r}\right),
$$

thus $\widehat{\mathrm{S}} \mathrm{al}$ is contractible (since, by Lemma 4.11, $\widehat{\mathrm{S}}_{+} \mathrm{l}_{+}\left(v_{r}\right)$ is contractible).

Let $\hat{f}$ be a path of $\widehat{\Gamma}$ beginning at $v_{0}$ and ending in $w$. Write $f=\rho(\hat{f})$. By Lemma 4.12, there exists an integer $r \geq 0$ and a positive path $g$ of $\Gamma(\mathscr{A})$ such that $f \sim\left(h_{A}\right)^{-r} g$. The lift of $\left(h_{A}\right)^{-r} g$ into $\widehat{\Gamma}$ beginning at $v_{0}$ has the form $\hat{h}_{1}^{-1} \cdots \hat{h}_{r}^{-1} \hat{g}$, where $\hat{g}$ is a positive path of $\widehat{\Gamma}$. By the definition of a cover of an oriented system, we have $\hat{f} \sim \hat{h}_{1}^{-1} \cdots \hat{h}_{r}^{-1} \hat{g}$, thus $\hat{g}$ begins at $v_{r}$ and ends in $w=\operatorname{end}(\hat{f})$, therefore $w \in V(\widehat{\Gamma})_{+}\left(v_{r}\right)$.

\section{REFERENCES}

[Br] K. S. Brown, Buildings, Springer-Verlag, New York, 1989.

[Co] R. Cordovil, On the homotopy of the Salvetti complexes determined by simplicial arrangements, preprint.

[De] P. Deligne, Les immeubles des groupes de tresses généralisés, Invent. Math. 17 (1972), 273302.

[FR] M. Falk and R. Randell, On the homotopy theory of arrangements, Complex Analytic Singularities, Advanced Studies in Pure Math., 8, North-Holland, Amsterdam, 1987, pp. 101124.

[JT] M. Jambu and H. Terao, Free arrangements of hyperplanes and supersolvable lattices, Adv. Math. 52 (1984), 248-258.

[Lj] E. S. Ljapin, Semigroups, Transl. Math. Monographs, vol. 3, Amer. Math. Soc., Providence, R.I., 1974.

[LW] A. T. Lundell and S. Weingram, The topology of CW complexes, Van Nostrand Reinhold, New York, 1969.

[Or] P. Orlik, Introduction to arrangements, CBMS Regional Conf. Ser. in Math., no. 72, Amer. Math. Soc., Providence, R.I., 1989.

[Pa1] L. Paris, The covers of a complexified real arrangement of hyperplanes and their fundamental groups, preprint.

[Pa2] - The Deligne complex of a real arrangement of hyperplanes, preprint.

[Pa3] _ Arrangements of hyperplanes with property $D$, preprint.

[Sa1] M. Salvetti, Topology of the complement of real hyperplanes in $\mathbb{C}^{N}$, Invent. Math. 88 (1987), 603-618.

[Sa2] _ On the homotopy theory of complexes associated to metrical-hemisphere complexes, preprint.

[Te1] H. Terao, Arrangements of hyperplanes and their freeness. I, II, J. Fac. Sci. Univ. Tokyo Sect. IA Math. 27 (1980), 293-320.

[Te2] _ Modular elements of lattices and topological fibration, Adv. Math. 62 (1986), 135-154.

[We] A. Weil, Sur les théorèmes de De Rham, Comment. Math. Helv. 26 (1952), 119-145.

Section de Mathématiques, Université de Genève, CP 240, CH-1211 Genève 24, SwitzerLAND

Department of Mathematics, University of Wisconsin, Madison, Wisconsin 53706

Current address: Département de Mathématiques, Université de Nantes, 2 vue de la Haussinière, 44072 Nantes, France 